\title{
Flexible Modeling of Variable Asymmetries in Cross-covariance Functions for Multivariate Random Fields
}

\author{
Ghulam A. Qadir ${ }^{1}$, Carolina Euán ${ }^{2}$ and Ying Sun ${ }^{1}$
}

\begin{abstract}
The geostatistical analysis of multivariate spatial data for inference as well as joint predictions (co-kriging) ordinarily relies on modeling of the marginal and cross-covariance functions. While the former quantifies the spatial dependence within variables, the latter quantifies the spatial dependence across distinct variables. The marginal covariance functions are always symmetric; however, the cross-covariance functions often exhibit asymmetries in the real data. Asymmetric cross-covariance implies change in the value of cross-covariance for interchanged locations on fixed order of variables. Such change of cross-covariance values are often caused due to the spatial delay in effect of the response of one variable on another variable. These spatial delays are common in environmental processes, especially when dynamic phenomena such as prevailing wind, ocean currents, etc., are involved. Here, we propose a novel approach to introduce flexible asymmetries in the cross-covariances of stationary multivariate covariance functions. The proposed approach involves modeling the phase component of the constrained cross-spectral features to allow for asymmetric crosscovariances. We show the capability of our proposed model to recover the cross-dependence structure and improve spatial predictions against traditionally used models through multiple simulation studies. Additionally, we illustrate our approach on a real trivariate dataset of particulate matter concentration $\left(\mathrm{PM}_{2.5}\right)$, wind speed and relative humidity. The real data example shows that our approach outperforms the traditionally used models, in terms of model fit and spatial predictions.
\end{abstract}

Some key words: Asymmetry; Coherence; Co-kriging; Matérn covariance; Phase spectrum.

\footnotetext{
${ }^{1}$ CEMSE Division, King Abdullah University of Science and Technology, Thuwal 23955-6900, Saudi Arabia. E-mail: ghulam.qadir@kaust.edu.sa; ying.sun@kaust.edu.sa

${ }^{2}$ Centro de Investigación en Matemáticas (CIMAT), Guanajuato, Mexico. E-mail: caroe@cimat.mx
} 


\section{Introduction}

The increasing availability of multivariate spatial datasets in recent decades as a result of technological advances in data collection have enticed researchers to jointly study multiple processes. An obvious advantage of multivariate modeling over univariate modeling is the utilization of cross-variable correlations for a superior statistical inference and more accurate predictions. Some real data examples where the cross-correlation between variables provides better understanding of the process include the spatial interpolation of trivariate pollutant data (Ozone $\left(\mathrm{O}_{3}\right)$, Sulphate $\left(\mathrm{SO}_{4}\right)$, Nitrate $\left.\left(\mathrm{NO}_{3}\right)\right)$ in Brown et al. (1994), joint modeling and spatial prediction of ozone and temperature data in Royle and Berliner (1999), and the joint modeling of conditional dependence in a trivariate soil elemental composition data (Arsenic, Iron, Chromium) in Guinness et al. (2014). For an advantageous multivariate spatial modeling, a sufficiently flexible multivariate covariance model that can adequately capture the nontrivial features of marginal and cross-covariances is imperative. Modeling of spatial processes commonly involves modeling the means and the covariances, but due to the requirement of the covariance matrix to be nonnegative definite, the modeling of the covariance is more challenging, and therefore, it is the main focus of this work.

Let $\mathbf{X}(\mathbf{s})=\left\{X_{1}(\mathbf{s}), \ldots, X_{p}(\mathbf{s})\right\}^{\mathrm{T}}$ be a zero mean $p$-variate random field indexed by $d-$ dimensional spatial locations $\mathbf{s} \in \mathbb{R}^{d}, d \geq 1$. We assume that the process $\mathbf{X}$ is secondorder stationary, and therefore the associated covariances $\operatorname{Cov}\{\mathbf{X}(\mathbf{s}), \mathbf{X}(\mathbf{s}+\mathbf{h})\}=\mathbf{C}(\mathbf{h})$ depend only on the spatial lag $\mathbf{h} \in \mathbb{R}^{d}$. The matrix-valued multivariate covariance function $\mathbf{C}(\mathbf{h})$ is then defined as $\mathbf{C}(\mathbf{h})=\left\{\mathrm{C}_{i j}(\mathbf{h})\right\}_{i, j=1}^{p}$, where $\mathrm{C}_{i j}(\mathbf{h})=E\left\{X_{i}(\mathbf{s}) X_{j}(\mathbf{s}+\mathbf{h})\right\}, i, j=$ $1, \ldots, p$. The marginal covariance function $\mathrm{C}_{i i}(\mathbf{h})$ quantifies the spatial dependence of the $i$ th process component $\left\{X_{i}(\mathbf{s}): \mathbf{s} \in \mathbb{R}^{d}\right\}, i=1, \ldots, p$, whereas the cross-covariance function $\mathrm{C}_{i j}(\mathbf{h}), i \neq j=1, \ldots, p$, quantifies the spatial dependence between the $i$ th and the $j$ th 
process components. For any set of spatial coordinates $\mathbf{s}_{1}, \ldots, \mathbf{s}_{n}$, and any positive integer $n$, the covariance matrix $\boldsymbol{\Sigma}$ associated with the random vector $\left\{\mathbf{X}\left(\mathbf{s}_{1}\right)^{\mathrm{T}}, \ldots, \mathbf{X}\left(\mathbf{s}_{n}\right)^{\mathrm{T}}\right\}^{\mathrm{T}} \in$ $\mathbb{R}^{n p}$ must be symmetric and nonnegative definite. An important feature of cross-covariance functions is that they are not necessarily symmetric. Specifically, for $i \neq j=1, \ldots, p$, and any two distinct arbitrary locations $\mathbf{s}$ and $\mathbf{s}^{\prime}$, the spatial dependence between $X_{i}(\mathbf{s})$ and $X_{j}\left(\mathbf{s}^{\prime}\right)$ is not necessarily equal to the spatial dependence between $X_{i}\left(\mathbf{s}^{\prime}\right)$ and $X_{j}(\mathbf{s})$. Therefore, if $\mathrm{C}_{i j}(\mathbf{h}) \neq \mathrm{C}_{j i}(\mathbf{h})$ (or equivalently $\mathrm{C}_{i j}(\mathbf{h}) \neq \mathrm{C}_{i j}(-\mathbf{h})$ ), then the pair of components $X_{i}$ and $X_{j}$ exhibit asymmetric spatial dependence, otherwise $X_{i}$ and $X_{j}$ exhibit symmetric spatial dependence. A challenging task is to construct a multivariate covariance model that allows for asymmetric cross-covariances without violating the nonnegative definiteness of $\boldsymbol{\Sigma}$.

The quest to develop valid and flexible models for $\mathbf{C}(\mathbf{h})$ has been underway for nearly three decades, which has led to a fairly rich literature on multivariate covariance models (see Genton and Kleiber (2015)). Some of the major works include the linear model of coregionalization (Goulard and Voltz, 1992; Schmidt and Gelfand, 2003; Wackernagel, 2003; Zhang, 2007), kernel convolution models (Ver Hoef and Barry, 1998; Ver Hoef, Cressie, and Barry, 2004), covariance convolution models (Gaspari and Cohn, 1999; Gaspari et al., 2006; Majumdar and Gelfand, 2007), separable or intrinsic models (Mardia and Goodall, 1993; Helterbrand and Cressie, 1994) and a very popular multivariate Matérn model (Gneiting, Kleiber, and Schlather, 2010; Apanasovich, Genton, and Sun, 2012). While all those models are highly valuable in multivariate spatial modeling, their original formulation is incapable of modeling the asymmetric spatial dependence between variables that may be present in the data. In such cases, the assumption of symmetric spatial dependence between variables disregards an important feature of the underlying process and might lead to less accurate spatial predictions (Ver Hoef and Cressie, 1993; Wackernagel, 2003).

A sizeable number of studies have been directed towards addressing the asymmetry fea- 
ture of the multivariate random fields over the last two decades. Li, Genton, and Sherman (2008) proposed a formal test of symmetry that can detect the presence of asymmetry in the cross-covariances of multivariate random fields. Sain and Cressie (2007) and Sain, Furrer, and Cressie (2011) introduced asymmetry in their Markov random field based model for multivariate lattice data. Apanasovich and Genton (2010) proposed latent dimensions based multivariate spatio-temporal cross-covariance models in which they introduced asymmetry by manoeuvring the latent dimensions associated with each variable. The conditional approach of multivariate modeling developed by Cressie and ZammitMangion (2016) specifies the asymmetric cross-covariances by using the asymmetric interaction functions. Christensen and Amemiya (2001) proposed a generalized shifted factor model that introduced asymmetry via lagged dependencies between latent factors and observed variables. Li and Zhang (2011) proposed a generic approach to introduce asymmetry in any valid stationary cross-covariances. Their idea is based on the fact that if $\mathbf{C}(\mathbf{h})=\left\{\mathrm{C}_{i j}(\mathbf{h})\right\}_{i, j=1}^{p}$ is any valid and stationary multivariate covariance function, then $\mathbf{C}^{\mathrm{LZ}}(\mathbf{h})=\left\{\mathbf{C}_{i j}^{\mathrm{LZ}}(\mathbf{h})\right\}_{i, j=1}^{p}=\left\{\mathrm{C}_{i j}\left(\mathbf{h}+\boldsymbol{\ell}_{i}-\boldsymbol{\ell}_{j}\right)\right\}_{i, j=1}^{p}, \boldsymbol{\ell}_{i} \in \mathbb{R}^{d}, i=1, \ldots, p$, is a valid multivariate covariance function that allows for asymmetry in the cross-covariances. The asymmetry is controlled by the vectors $\boldsymbol{\ell}_{i}, i=1, \ldots, p$, which introduces delay in the cross-covariances such that the $\mathbf{C}_{i j}^{\mathrm{LZ}}(\cdot)$ attains its extreme value $\left(\max \left\{\left|\mathbf{C}_{i j}^{\mathrm{LZ}}(\cdot)\right|\right\}\right)$ at $\mathbf{h}=\boldsymbol{\ell}_{j}-\boldsymbol{\ell}_{i}$ instead of $\mathbf{h}=\mathbf{0}$.

Although Li and Zhang's approach for modeling asymmetric cross-covariances is very useful and flexible for bivariate $(p=2)$ random fields, it is not sufficiently flexible for modeling variable asymmetries for the cases where $p>2$. This insufficiency stems from the fact that there are only $p-1$ non-redundant asymmetry parameters $\boldsymbol{\ell}_{i}$ to model asymmetric spatial dependence between $p ! /\{2 !(p-2) !\}$ pairs of variables. Therefore, for a specified asymmetric behavior in any $p-1$ pairs of variables, the asymmetry within the remaining $\{(p-$ 
1) $(p-2)\} / 2$ pairs of variables cannot be established independently. A similar limitation exists in the Apanasovich and Genton (2010)'s purely spatial asymmetric multivariate covariance model.

In this work, we propose an approach to flexibly introduce asymmetry in cross-covariances of stationary multivariate covariance functions such that the asymmetric spatial dependence between every pair of variables can be controlled independently by unique $p ! /\{2 !(p-2) !\}$ vector-valued asymmetry parameters. While the proposed approach enjoys flexible specification of asymmetry, it requires constraining the absolute coherence functions, which in turn, limits the allowed maximal absolute cross-correlations. We provide sufficient conditions for the validity of our proposed class of asymmetric model for trivariate random fields, and compare our model's performance with Li and Zhang's model.

In Section 2, we explain our proposed class of asymmetric models through its development in the spectral domain. We evaluate our model's performance through multiple simulation studies in Section 3, in which we also shed light upon the limitations of Li and Zhang's model by using examples of cases where it is inadequate for modeling asymmetric spatial dependence. In Section 4, we illustrate the application of our proposed asymmetric model on a trivariate dataset of $\mathrm{PM}_{2.5}$, wind speed and relative humidity while comparing its performance with Li and Zhang's model and symmetric models. In Section 5, we provide a brief discussion and potential future extensions of our work.

\section{Asymmetric Models and Inference}

The multivariate covariance function $\mathbf{C}(\mathbf{h})=\left\{\mathrm{C}_{i j}(\mathbf{h})\right\}_{i, j=1}^{p}$ associated with second-order stationary multivariate process $\mathbf{X}(\mathbf{s})=\left\{X_{1}(\mathbf{s}), \ldots, X_{p}(\mathbf{s})\right\}^{\mathrm{T}}$ satisfies the inequality $\left|\mathrm{C}_{i j}(\mathbf{h})\right|^{2} \leq$ $\mathrm{C}_{i i}(\mathbf{0}) \mathrm{C}_{j j}(\mathbf{0}), i, j=1, \ldots, p$. However, it need not satisfy the inequality $\left|\mathrm{C}_{i j}(\mathbf{h})\right| \leq$ $\mathrm{C}_{i j}(\mathbf{0}), i \neq j$, because the maximum of $\left|\mathrm{C}_{i j}(\mathbf{h})\right|$ is not restricted at $\mathbf{h}=\mathbf{0}$. Let us sup- 
pose that $\arg \max _{\mathbf{h}}\left|\mathrm{C}_{i j}(\mathbf{h})\right|=\boldsymbol{\ell}_{i j}, \boldsymbol{\ell}_{i j}=-\boldsymbol{\ell}_{j i}, i \neq j$, then $X_{j}(\mathbf{s})$ exhibit the strongest crosscovariance with $X_{i}\left(\mathbf{s}-\boldsymbol{\ell}_{i j}\right)$ instead of $X_{i}(\mathbf{s})$, and $\boldsymbol{\ell}_{i j}$ is the spatial delay in reaction of variable $X_{i}$ to the fluctuations in variable $X_{j}$, which leads to asymmetric cross-covariance in light of the delay effect (Wackernagel, 2003). Such delayed effect processes are commonly observed in atmospheric sciences (Gelfand et al., 2010, .p 497) where the vector $\boldsymbol{\ell}_{i j}$ can be associated with the direction of prevailing weather fronts. For example, consider a bivariate process where $X_{1}$ is the traffic emissions and $X_{2}$ is the ambient air pollution concentration, then $\boldsymbol{\ell}_{12}$ might be related to the predominant wind pattern in the region, so that pollution at site s, $X_{2}(\mathbf{s})$, is more correlated with emissions downwind, $X_{1}\left(\mathbf{s}-\ell_{12}\right)$, than at the site $\mathbf{s}, X_{1}(\mathbf{s})$. In practice, when we have more than two variables, complex interplay of variables with dynamic phenomena such as prevailing weather fronts, can lead to different spatial delays $\boldsymbol{\ell}_{i j}$ for each pair of the variables. To this end, we propose a class of asymmetric model which allows for independent spatial delays for each pair of variable, the theoretical development of which is described Sections 2.1-2.2.

\subsection{Spectral Formulation and Model Construction}

The validity of $\mathbf{C}(\mathbf{h})$ is generally ensured by utilizing the much celebrated Cramér's Theorem (Cramér, 1940) in its spectral density version (Wackernagel, 2003, p. 215; Kleiber, 2017) which states that: The necessary and sufficient condition for the matrix valued function $\mathbf{C}: \mathbb{R}^{d} \rightarrow \mathbb{C}^{p \times p}, \mathbf{C}(\mathbf{h})=\left\{\mathrm{C}_{i j}(\mathbf{h})\right\}_{i, j=1}^{p}$, to be nonnegative definite is its representation as $\mathrm{C}_{i j}(\mathbf{h})=\int_{\mathbb{R}^{d}} \exp \left(\imath \boldsymbol{\omega}^{\mathrm{T}} \mathbf{h}\right) f_{i j}(\boldsymbol{\omega}) \mathrm{d} \boldsymbol{\omega}, \quad(\imath=\sqrt{-1}), i, j=1, \ldots, p$, where the matrix $\mathbf{f}(\boldsymbol{\omega})=$ $\left\{f_{i j}(\boldsymbol{\omega})\right\}_{i, j=1}^{p}$ is nonnegative definite for all $\boldsymbol{\omega} \in \mathbb{R}^{d}$.

The matrix $\mathbf{f}(\boldsymbol{\omega})$ is a Hermitian matrix for all $\boldsymbol{\omega} \in \mathbb{R}^{d}$, such that its diagonal entries are defined by the spectral densities $f_{i i}(\boldsymbol{\omega})$ of the marginal covariance functions $\mathrm{C}_{i i}(\mathbf{h}), \quad i=$ $1, \ldots, p$, and the off-diagonal entries are defined by the cross-spectral densities $f_{i j}(\boldsymbol{\omega})$ of the 
cross-covariance functions $\mathrm{C}_{i j}(\mathbf{h}), i \neq j=1, \ldots, p$.

In general, the cross-spectral densities $f_{i j}(\boldsymbol{\omega})$ can be complex-valued with a polar form notation $f_{i j}(\boldsymbol{\omega})=\alpha_{i j}(\boldsymbol{\omega}) \exp \left\{\imath \Phi_{i j}(\boldsymbol{\omega})\right\}$, where $\alpha_{i j}(\boldsymbol{\omega})=\left|f_{i j}(\boldsymbol{\omega})\right| \equiv \sqrt{\operatorname{Re}\left\{f_{i j}(\boldsymbol{\omega})\right\}^{2}+\operatorname{Im}\left\{f_{i j}(\boldsymbol{\omega})\right\}^{2}}$ and $\Phi_{i j}(\boldsymbol{\omega})=\arg f_{i j}(\boldsymbol{\omega}) \equiv \tan ^{-1}\left[\operatorname{Im}\left\{f_{i j}(\boldsymbol{\omega})\right\} / \operatorname{Re}\left\{f_{i j}(\boldsymbol{\omega})\right\}\right]$ are called the cross-amplitude spectrum and the phase spectrum, respectively (Priestley, 1981). Since the matrix $\mathbf{f}(\boldsymbol{\omega})$ is a Hermitian matrix, the relations $\alpha_{i j}(\boldsymbol{\omega})=\alpha_{j i}(\boldsymbol{\omega})$ and $\Phi_{i j}(\boldsymbol{\omega})=-\Phi_{j i}(\boldsymbol{\omega})$ always hold true for all $\boldsymbol{\omega} \in \mathbb{R}^{d}$. When a cross-spectral density $f_{i j}(\boldsymbol{\omega})$ is real-valued for all $\boldsymbol{\omega} \in \mathbb{R}^{d}$, or equivalently $\Phi_{i j}: \mathbb{R}^{d} \rightarrow\{\pi(k \pm 1), k \in \mathbb{Z}\}$, the corresponding cross-covariance $\mathrm{C}_{i j}(\mathbf{h})$ is an even function $\left(\mathrm{C}_{i j}(\mathbf{h})=\mathrm{C}_{i j}(-\mathbf{h})\right)$ (Wackernagel, 2003, p.153), implying symmetric spatial dependence between $X_{i}$ and $X_{j}$. Thus, introducing asymmetry can be considered equivalent to modifying the phase spectrum of the corresponding real-valued cross-spectral densities such that the new cross-spectral densities become complex-valued. However, the modification should be done with due diligence such that the modified complex-valued matrix of spectral densities $\tilde{\mathbf{f}}(\boldsymbol{\omega})=\left\{\tilde{f}_{i j}(\boldsymbol{\omega})\right\}_{i, j=1}^{p}$ is Hermitian nonnegative definite for all $\boldsymbol{\omega} \in \mathbb{R}^{d}$. Specifically, let $\ddot{\mathbf{f}}(\boldsymbol{\omega})=\left\{\ddot{f}_{i j}(\boldsymbol{\omega})\right\}_{i, j=1}^{p}$ be the real-valued matrix of spectral densities for a valid stationary matrix-valued multivariate covariance function $\mathbf{C}(\mathbf{h})=\left\{\mathrm{C}_{i j}(\mathbf{h})\right\}_{i, j=1}^{p}$ with symmetric cross-covariances $\mathrm{C}_{i j}(\mathbf{h}), i \neq j=1, \ldots, p$. The real-valued cross-spectral densities $\ddot{f}_{i j}(\boldsymbol{\omega})$ takes the form $\ddot{f}_{i j}(\boldsymbol{\omega})=\left|\ddot{f}_{i j}(\boldsymbol{\omega})\right| \exp \left\{\imath \ddot{\Phi}_{i j}(\boldsymbol{\omega})\right\}$, where $\ddot{\Phi}_{i j}: \mathbb{R}^{d} \rightarrow\{\pi(k \pm 1), k \in \mathbb{Z}\}$ and $\ddot{\Phi}_{i j}(\boldsymbol{\omega})=-\ddot{\Phi}_{j i}(\boldsymbol{\omega})$ for all $\boldsymbol{\omega} \in \mathbb{R}^{d}$. We define $\tilde{\mathbf{f}}(\boldsymbol{\omega})$ as $\tilde{f}_{i i}(\boldsymbol{\omega})=\ddot{f}_{i i}(\boldsymbol{\omega}), i=1, \ldots, p$, and $\tilde{f}_{i j}(\boldsymbol{\omega})=\left|\ddot{f}_{i j}(\boldsymbol{\omega})\right| \exp \left[\imath\left\{\ddot{\Phi}_{i j}(\boldsymbol{\omega})+\dot{\Phi}_{i j}(\boldsymbol{\omega})\right\}\right], i \neq j=1, \ldots, p$, where $\dot{\Phi}_{i j}(\boldsymbol{\omega})$ is a function of the frequency $\boldsymbol{\omega}$ such that $\dot{\Phi}_{i j}(\boldsymbol{\omega})=-\dot{\Phi}_{j i}(\boldsymbol{\omega})$ for all $\boldsymbol{\omega} \in \mathbb{R}^{d}$. The phase spectrum for the modified cross-spectral density $\tilde{f}_{i j}(\boldsymbol{\omega})$ is $\tilde{\Phi}_{i j}(\boldsymbol{\omega})=\ddot{\Phi}_{i j}(\boldsymbol{\omega})+\dot{\Phi}_{i j}(\boldsymbol{\omega})$.

One valid specification for $\dot{\Phi}_{i j}(\boldsymbol{\omega}), i \neq j=1, \ldots, p$, is $\dot{\Phi}_{i j}(\boldsymbol{\omega})=\phi_{i}(\boldsymbol{\omega})-\phi_{j}(\boldsymbol{\omega})$, where $\phi_{i}(\boldsymbol{\omega}), i=1, \ldots, p$, are any scalar functions of the frequency $\boldsymbol{\omega}$. Then $\tilde{\mathbf{f}}(\boldsymbol{\omega})$ can be decomposed as a Hadamard product of the two nonnegative definite matrices $\tilde{\mathbf{f}}(\boldsymbol{\omega})=\ddot{\mathbf{f}}(\boldsymbol{\omega}) \circ \mathbf{P}(\boldsymbol{\omega})$, 
where $\mathbf{P}(\boldsymbol{\omega})=\mathbf{p}(\boldsymbol{\omega}) \mathbf{p}(\boldsymbol{\omega})^{*}, \mathbf{p}(\boldsymbol{\omega})=\left[\exp \left\{\imath \phi_{1}(\boldsymbol{\omega})\right\}, \ldots, \exp \left\{\imath \phi_{p}(\boldsymbol{\omega})\right\}\right]^{\mathrm{T}}$, “*” denotes conjugate transpose, and the nonnegative definiteness of $\tilde{\mathbf{f}}(\boldsymbol{\omega})$ is then guaranteed by the Schur product theorem (Horn and Johnson, 2013, Theorem 7.5.3). This formulation is considered in the spectral model of Guinness et al. (2014), and in fact, the Li and Zhang's asymmetric model also fits in the aforementioned formulation with $\phi_{i}(\boldsymbol{\omega})=\boldsymbol{\omega}^{\mathrm{T}} \boldsymbol{\ell}_{i}, i=1, \ldots, p$, which is a linear transformation of the frequency $\boldsymbol{\omega}$ with the asymmetry parameter $\boldsymbol{\ell}_{i}$.

However, this formulation might not be flexible enough to model asymmetric spatial dependence between variables in the cases where $p>2$. For example, consider a trivariate case where we specify $\phi_{1}(\boldsymbol{\omega})=0$ for all $\boldsymbol{\omega} \in \mathbb{R}^{d}$, and any arbitrary flexible functions for $\phi_{2}: \mathbb{R}^{d} \rightarrow \mathbb{R}$ and $\phi_{3}: \mathbb{R}^{d} \rightarrow \mathbb{R}$. Then $\dot{\Phi}_{12}(\cdot)=-\phi_{2}(\cdot)$ and $\dot{\Phi}_{13}(\cdot)=-\phi_{3}(\cdot)$ are specified to be any flexible functions, but $\dot{\Phi}_{23}(\cdot)$ is forced to be the difference of $\phi_{2}(\cdot)$ and $\phi_{3}(\cdot)$, i.e., $\dot{\Phi}_{23}(\cdot)=\phi_{2}(\cdot)-\phi_{3}(\cdot)$. To construct a flexible model for asymmetric cross-covariances, we avoid the restricted form $\dot{\Phi}_{i j}(\boldsymbol{\omega})=\phi_{i}(\boldsymbol{\omega})-\phi_{j}(\boldsymbol{\omega})$ and allow for a general $\dot{\Phi}_{i j}(\boldsymbol{\omega})$ by establishing the validity conditions.

Theorem 1. Let the symmetric real-valued matrix of spectral and cross-spectral densities $\ddot{\mathbf{f}}(\boldsymbol{\omega})=\left\{\ddot{f}_{i j}(\boldsymbol{\omega})\right\}_{i, j=1}^{p}, \quad p \leq 3$, be nonnegative definite for all $\boldsymbol{\omega} \in \mathbb{R}^{d}$, and let the modified $\operatorname{matrix} \tilde{\mathbf{f}}(\boldsymbol{\omega})=\left\{\tilde{f}_{i j}(\boldsymbol{\omega})\right\}_{i, j=1}^{p}=\ddot{\mathbf{f}}(\boldsymbol{\omega}) \circ \dot{\mathbf{P}}(\boldsymbol{\omega})$, where $\dot{\mathbf{P}}(\boldsymbol{\omega})=\left[\exp \left\{\imath \dot{\Phi}_{i j}(\boldsymbol{\omega})\right\}\right]_{i, j=1}^{p}$ such that $\dot{\Phi}_{i j}(\boldsymbol{\omega})=-\dot{\Phi}_{j i}(\boldsymbol{\omega}), i, j=1, \ldots, p$. Then for any general $\dot{\Phi}_{i j}(\boldsymbol{\omega})$, the Hermitian matrix $\tilde{\mathbf{f}}(\boldsymbol{\omega})$ is nonnegative definite for all $\boldsymbol{\omega} \in \mathbb{R}^{d}$ if the associated coherence functions $\ddot{\gamma}_{i j}(\boldsymbol{\omega})=$ $\ddot{f}_{i j}(\boldsymbol{\omega}) /\left[\left\{\ddot{f}_{i i}(\boldsymbol{\omega}) \ddot{f}_{j j}(\boldsymbol{\omega})\right\}^{1 / 2}\right], i, j=1, \ldots, p$, satisfy $\sum_{i<j}^{p}\left|\ddot{\gamma}_{i j}(\boldsymbol{\omega})\right|^{2} \leq 1-2 \prod_{i<j}^{p}\left|\ddot{\gamma}_{i j}(\boldsymbol{\omega})\right|$ for all $\omega \in \mathbb{R}^{d}$.

Moreover, for a particular choice of $\dot{\Phi}_{i j}(\boldsymbol{\omega})=-\boldsymbol{\omega}^{\mathrm{T}} \boldsymbol{\ell}_{i j}, \quad \boldsymbol{\ell}_{i j}=-\boldsymbol{\ell}_{j i} \in \mathbb{R}^{d}, \quad i, j=$ $1, \ldots, p, p \leq 3$, we propose a new class of flexible asymmetric cross-covariance models.

Theorem 2. Let $\mathbf{C}(\mathbf{h})=\left\{\mathrm{C}_{i j}(\mathbf{h})\right\}_{i, j=1}^{p}, \quad p \leq 3, \mathbf{h} \in \mathbb{R}^{d}$ be any valid stationary matrix- 
valued multivariate covariance function with the associated coherence functions $\ddot{\gamma}_{i j}(\boldsymbol{\omega}), i, j=$ $1, \ldots, p$. Then for any $\boldsymbol{\ell}_{i j}=-\boldsymbol{\ell}_{j i} \in \mathbb{R}^{d}, i, j=1, \ldots, p$, the multivariate covariance function $\mathbf{C}^{\mathrm{a}}(\mathbf{h})=\left\{\mathrm{C}_{i j}^{\mathrm{a}}(\mathbf{h})\right\}_{i, j=1}^{p}=\left\{\mathrm{C}_{i j}\left(\mathbf{h}-\boldsymbol{\ell}_{i j}\right)\right\}_{i, j=1}^{p}, \quad p \leq 3$, with asymmetric cross-covariances $\mathrm{C}_{i j}^{\mathrm{a}}(\mathbf{h}), i \neq j=1, \ldots, p$, is valid if $\sum_{i<j}^{p}\left|\ddot{\gamma}_{i j}(\boldsymbol{\omega})\right|^{2} \leq 1-2 \prod_{i<j}^{p}\left|\ddot{\gamma}_{i j}(\boldsymbol{\omega})\right|$ for all $\boldsymbol{\omega} \in \mathbb{R}^{d}$.

The proposed class of multivariate covariance $\mathbf{C}^{\mathrm{a}}(\mathbf{h})$ with asymmetric cross-covariances $\mathrm{C}_{i j}^{\mathrm{a}}(\mathbf{h}), i \neq j=1, \ldots, p, \quad p \leq 3$, can allow asymmetry in any stationary multivariate covariance model. In principle, the validity condition based on the coherence functions given in Theorem 2 can be easily satisfied by constraining some model specific parameters of the stationary multivariate covariance model. Here we provide such conditions on model parameters for some popular multivariate covariance models such as the flexible multivariate Matérn model (Apanasovich, Genton, and Sun, 2012), parsimonious multivariate Matérn model (Gneiting, Kleiber, and Schlather, 2010), and separable or intrinsic model (Mardia and Goodall, 1993) in Corollary 2.1-2.3. Similar model specific parameter constraints can be derived easily for any other stationary multivariate covariance model.

Corollary 2.1. Let $\mathbf{C}(\mathbf{h})=\left\{\mathrm{C}_{i j}(\mathbf{h})\right\}_{i, j=1}^{p}, p \leq 3$ be a flexible multivariate Matérn model (Apanasovich, Genton, and Sun, 2012), i.e., $\mathrm{C}_{i j}(\mathbf{h})=\rho_{i j} \sigma_{i} \sigma_{j} \mathrm{M}\left(\mathbf{h} \mid \nu_{i j}, a_{i j}\right)$, where $\mathrm{M}(\mathbf{h} \mid \nu, a)$ is a Matérn correlation function with spatial scale $a>0$ and smoothness $\nu>0, \sigma_{i}>0$ is the marginal standard deviation, and $\rho_{i j}$ is the colocated correlation coefficient. The parameters are constrained such that $(1) \nu_{i j}=\left(\nu_{i i}+\nu_{j j}\right) / 2+\Delta_{A}\left(1-A_{i j}\right), i, j=1, \ldots, p$, where $0 \leq$ $A_{i j} \leq 1$ form a valid correlation matrix, $\Delta_{A} \geq 0 ;(2)\left(-a_{i j}^{2}\right)_{i, j=1}^{p}$ form a conditional nonnegative definite matrix; and (3) $\rho_{i j}=\beta_{i j} a_{i j}^{-2 \Delta_{A}-\nu_{i i}-\nu_{j j}}\left[\Gamma\left\{\left(\nu_{i i}+\nu_{j j}\right) / 2+d / 2\right\} \Gamma\left(\nu_{i j}\right)\right] / \Gamma\left(\nu_{i j}+d / 2\right)$ where $\beta_{i j}$ form a valid correlation matrix. Then for any $\boldsymbol{\ell}_{i j}=-\boldsymbol{\ell}_{j i} \in \mathbb{R}^{d}, i, j=1, \ldots, p$, the multivariate covariance function $\mathbf{C}^{\mathrm{a}}(\mathbf{h})=\left\{\mathrm{C}_{i j}^{\mathrm{a}}(\mathbf{h})\right\}_{i, j=1}^{p}=\left\{\mathrm{C}_{i j}\left(\mathbf{h}-\boldsymbol{\ell}_{i j}\right)\right\}_{i, j=1}^{p}, p \leq 3$, is valid if $\sum_{i<j}^{p}\left|\beta_{i j}\right|^{2} \leq 1-2 \prod_{i<j}^{p}\left|\beta_{i j}\right|$. 
Corollary 2.2. Let $\mathbf{C}(\mathbf{h})=\left\{\mathrm{C}_{i j}(\mathbf{h})\right\}_{i, j=1}^{p}, p \leq 3$, be a parsimonious multivariate Matérn model (Gneiting, Kleiber, and Schlather, 2010), i.e., $\mathrm{C}_{i j}(\mathbf{h})=\rho_{i j} \sigma_{i} \sigma_{j} \mathrm{M}\left\{\mathbf{h} \mid\left(\nu_{i i}+\nu_{j j}\right) / 2, a\right\}$, $\rho_{i j}=\beta_{i j}\left[\sqrt{\Gamma\left(\nu_{i i}+d / 2\right) \Gamma\left(\nu_{j j}+d / 2\right)} \Gamma\left\{\left(\nu_{i i}+\nu_{j j}\right) / 2\right\}\right] /\left[\sqrt{\Gamma\left(\nu_{i i}\right) \Gamma\left(\nu_{j j}\right)} \Gamma\left\{\left(\nu_{i i}+\nu_{j j}\right) / 2+d / 2\right\}\right]$. Then for any $\boldsymbol{\ell}_{i j}=-\boldsymbol{\ell}_{j i} \in \mathbb{R}^{d}, i, j=1, \ldots, p$, the multivariate covariance function $\mathbf{C}^{\mathrm{a}}(\mathbf{h})=$ $\left\{\mathrm{C}_{i j}^{\mathrm{a}}(\mathbf{h})\right\}_{i, j=1}^{p}=\left\{\mathrm{C}_{i j}\left(\mathbf{h}-\boldsymbol{\ell}_{i j}\right)\right\}_{i, j=1}^{p}, p \leq 3$, is valid if $\sum_{i<j}^{p}\left|\beta_{i j}\right|^{2} \leq 1-2 \prod_{i<j}^{p}\left|\beta_{i j}\right|$.

Corollary 2.3. Let $\mathbf{C}(\mathbf{h})=\mathbf{R} \rho(\mathbf{h})$ be a separable or intrinsic model (Mardia and Goodall, 1993) where $\mathbf{R}=\left(r_{i j}\right)_{i, j=1}^{p}, \quad p \leq 3$, is a symmetric positive definite matrix and $\rho(\mathbf{h})$ is any valid stationary correlation function. Then for any $\boldsymbol{\ell}_{i j}=-\boldsymbol{\ell}_{j i} \in \mathbb{R}^{d}, i, j=1, \ldots, p$, the multivariate covariance function $\mathbf{C}^{\mathrm{a}}(\mathbf{h})=\left\{\mathrm{C}_{i j}^{\mathrm{a}}(\mathbf{h})\right\}_{i, j=1}^{p}=\left\{r_{i j} \rho\left(\mathbf{h}-\boldsymbol{\ell}_{i j}\right)\right\}_{i, j=1}^{p}, p \leq 3$, is valid if $\sum_{i<j}^{p}\left|r_{i j} /\left\{\left(r_{i i} r_{j j}\right)^{1 / 2}\right\}\right|^{2} \leq 1-2 \prod_{i<j}^{p}\left|r_{i j} /\left\{\left(r_{i i} r_{j j}\right)^{1 / 2}\right\}\right|$.

The sufficient condition truncates the original parameter space of the absolute coherence functions of the corresponding symmetric model, and we visualize this truncation in Figure 1. The green dome-like shape shown in Figure 1(a) represents the parameter space of absolute coherence functions $\left|\ddot{\gamma}_{i j}(\boldsymbol{\omega})\right|$, at any frequency $\boldsymbol{\omega} \in \mathbb{R}^{d}$, that guarantees the validity of $\mathbf{C}^{\mathrm{a}}(\mathbf{h})$, whereas the blue cone-like shape represents the parameter space that guarantees the validity of the corresponding symmetric model $\mathbf{C}(\mathbf{h})$. Any point inside the the green dome-like shape leads to a valid $\mathbf{C}^{\mathrm{a}}(\mathbf{h})$, whereas any point inside the the blue cone-like shape leads to a valid $\mathbf{C}(\mathbf{h})$. Additionally, Figure 1(b) and Figure 1(c) show the upper bound of $\left|\ddot{\gamma}_{23}(\boldsymbol{\omega})\right|$ over all possible values of $\left(\left|\ddot{\gamma}_{12}(\boldsymbol{\omega})\right|,\left|\ddot{\gamma}_{13}(\boldsymbol{\omega})\right|\right)$, for $\mathbf{C}^{\mathrm{a}}(\mathbf{h})$ and $\mathbf{C}(\mathbf{h})$, respectively. Similarly, Figure 1(e) and Figure 1(f) show the lower bound of $\left|\ddot{\gamma}_{23}(\boldsymbol{\omega})\right|$ for $\mathbf{C}^{\mathrm{a}}(\mathbf{h})$ and $\mathbf{C}(\mathbf{h})$, respectively. The white region in Figures 1(b) and 1(e) highlight the truncated parameter space where Theorems 1-2 do not satisfy. Furthermore, we show the reduction in the upper bound and the lower bound of $\left|\ddot{\gamma}_{23}(\boldsymbol{\omega})\right|$ as we move to $\mathbf{C}^{\mathrm{a}}(\mathbf{h})$ from $\mathbf{C}(\mathbf{h})$ in Figure $1(\mathrm{~d})$ and Figure $1(\mathrm{~g})$, respectively. The lower bound of $\left|\ddot{\gamma}_{23}(\boldsymbol{\omega})\right|$ in the untruncated space remains unchanged as we 
move to $\mathbf{C}^{\mathrm{a}}(\mathbf{h})$ from $\mathbf{C}(\mathbf{h})$ because it is always zero for both the models in the region where Theorems 1-2 satisfy. However, moving from $\mathbf{C}(\mathbf{h})$ to $\mathbf{C}^{\mathrm{a}}(\mathbf{h})$ exhibits large reduction in the upper bound of $\left|\ddot{\gamma}_{23}(\boldsymbol{\omega})\right|$ in regions where both $\left|\ddot{\gamma}_{12}(\boldsymbol{\omega})\right|$ and $\left|\ddot{\gamma}_{13}(\boldsymbol{\omega})\right|$ are simultaneously high.

The visualization reveals that the proposed model does not allow the three process components $X_{i}, i=1,2,3$, to be concurrently highly coherent to each other at any frequency $\boldsymbol{\omega} \in \mathbb{R}^{d}$. The triplet $\left(\left|\ddot{\gamma}_{12}(\boldsymbol{\omega})\right|,\left|\ddot{\gamma}_{13}(\boldsymbol{\omega})\right|,\left|\ddot{\gamma}_{23}(\boldsymbol{\omega})\right|\right)$ which simultaneously maximizes all the three absolute coherence functions while satisfying the sufficient condition for the validity of $\mathbf{C}^{\mathrm{a}}(\mathbf{h})$ is $(0.5,0.5,0.5)$ (shown as a red point Figure $\left.1(\mathrm{~b})\right)$, whereas the value of such a triplet is $(1,1,1)$ (shown as a red point Figure $1(\mathrm{c})$ ) for the corresponding symmetric model $\mathbf{C}(\mathbf{h})$. For a bivariate $(p=2)$ case, the sufficient condition in Theorem 1 and Theorem 2 is always satisfied as the inequality $\left|\ddot{\gamma}_{12}(\boldsymbol{\omega})\right|^{2} \leq 1$ for all $\boldsymbol{\omega} \in \mathbb{R}^{d}$, holds true for all the valid stationary bivariate covariance models, and consequently, for the bivariate case, the proposed asymmetric model $\mathbf{C}^{\mathrm{a}}(\mathbf{h})$ is exactly equivalent to the Li and Zhang's asymmetric model $\mathbf{C}^{\mathrm{LZ}}(\mathbf{h})$ with $\ell_{1}=0$ and $\ell_{12}=\ell_{2}$. Therefore, the truncation of the original parameter space of absolute coherence functions occurs only in the trivariate case. This truncation may seem restrictive and can be viewed as the limitation of the proposed construction, however, for a trivariate case, the inclusion of the asymmetry parameters $\boldsymbol{\ell}_{i j}, i, j=1,2,3$, for separately modeling the asymmetry between every pair of variables, brings a significant amount of flexibility in the proposed class of asymmetric models.

The asymmetry parameter $\boldsymbol{\ell}_{i j}$ has a straightforward interpretation as it shifts the entire cross-covariance structure of the corresponding symmetric $\mathrm{C}_{i j}(\cdot), i \neq j=1, \ldots, p$, away from the origin such that the extreme cross-covariance value is now centered at $\boldsymbol{\ell}_{i j}$, and since $\boldsymbol{\ell}_{i j}=-\boldsymbol{\ell}_{j i}$, the corresponding symmetric $\mathrm{C}_{j i}(\cdot)$ is re-centered at $-\boldsymbol{\ell}_{i j}$. Since $\boldsymbol{\ell}_{i j}=-\boldsymbol{\ell}_{j i} \in$ $\mathbb{R}^{d}, i, j=1, \ldots, 3$, the marginal covariance functions $\mathbf{C}_{i i}^{\mathrm{a}}(\mathbf{h})=\mathrm{C}_{i i}\left(\mathbf{h}-\boldsymbol{\ell}_{i i}\right)$ will always be symmetric as $\boldsymbol{\ell}_{i i}=\mathbf{0}, i=1, \ldots, p$. Figures $2(\mathrm{~g})-2(\mathrm{i})$ show one example of the proposed 
asymmetric cross-covariances $\mathbf{C}_{12}^{\mathrm{a}}(\mathbf{h})=\mathrm{C}_{12}\left(\mathbf{h}-\boldsymbol{\ell}_{12}\right), \mathbf{C}_{13}^{\mathrm{a}}(\mathbf{h})=\mathrm{C}_{13}\left(\mathbf{h}-\boldsymbol{\ell}_{13}\right)$ and $\mathbf{C}_{23}^{\mathrm{a}}(\mathbf{h})=$ $\mathrm{C}_{23}\left(\mathbf{h}-\boldsymbol{\ell}_{23}\right)$, respectively, as a function of spatial lags $\mathbf{h}=\left(h_{1}, h_{2}\right),-0.6 \leq h_{1}, h_{2} \leq 0.6$, for a trivariate process with asymmetry parameters $\ell_{12}=\ell_{13}=\ell_{23}=(-0.15,-0.15)$. This example of an asymmetric cross-covariance function is later used in our simulation study in Section 3 and a detailed parameter specification of this example is mentioned in Section 3.1. The specified asymmetry parameters in this example re-centers all the corresponding symmetric cross-covariances $\mathrm{C}_{12}(\cdot), \mathrm{C}_{13}(\cdot)$ and $\mathrm{C}_{23}(\cdot)$ away from the origin $\mathbf{h}=\mathbf{0}$ such that the extreme value is attained at lag $\mathbf{h}=(-0.15,-0.15)$ instead of $\mathbf{h}=\mathbf{0}$, thus signifying the asymmetric spatial dependence between all the three pairs of variables. The advantage of our model is that the asymmetric behavior for one pair of variables does not affect the asymmetric behavior of the other pairs. The illustrated cross-covariances in Figures 2(g)-2(i) is an example which cannot be comprehended with any other existing class of asymmetric trivariate covariance model.

\subsection{Parameter estimation}

Let $\boldsymbol{\theta}^{\mathrm{a}}=\left\{\boldsymbol{\theta}_{\mathrm{m}}, \boldsymbol{\theta}_{\mathrm{c}}, \boldsymbol{\theta}_{\mathrm{s}}\right\}$ be the set of parameters that fully specify the proposed asymmetric multivariate covariance model $\mathbf{C}^{\mathrm{a}}(\mathbf{h})=\left\{\mathrm{C}_{i j}\left(\mathbf{h}-\boldsymbol{\ell}_{i j}\right)\right\}_{i, j=1}^{p}$, where $\boldsymbol{\theta}_{\mathrm{m}}$ denotes the set of parameters that defines the marginal covariance functions $\mathrm{C}_{i i}(\mathbf{h}), i=1, \ldots, p, \quad \boldsymbol{\theta}_{\mathrm{c}}$ denotes the set of parameters that defines the corresponding symmetric cross-covariance functions $\mathrm{C}_{i j}(\mathbf{h}), i \neq j=1, \ldots, p$, and $\boldsymbol{\theta}_{\mathrm{s}}$ denotes the set of asymmetry parameters $\ell_{i j}, i<j=1, \ldots, p$, for $p \leq 3$. Clearly, only $p ! /\{2 !(p-2) !\}$ vector-valued asymmetry parameters are required to specify $\mathbf{C}^{\mathrm{a}}(\mathbf{h})$ as $\boldsymbol{\ell}_{i i}=\mathbf{0}, i=1, \ldots, p$, and $\boldsymbol{\ell}_{j i}=-\boldsymbol{\ell}_{i j}, i<j=1, \ldots, p$. We assume that $\mathbf{X}(\mathbf{s})=\left\{X_{1}(\mathbf{s}), \ldots, X_{p}(\mathbf{s})\right\}^{\mathrm{T}}, \mathbf{s} \in \mathbb{R}^{d}, d \geq 1$ is a zero mean $p$-variate stationary Gaussian random field and estimate model parameters by likelihood maximization. The Gaussian maximum likelihood estimation (MLE), in general, is a challenging problem 
when number of spatial locations are large, and more so in the multivariate spatial data as multiple process components lead to large covariance matrix. The difficulty mainly lies in the Cholesky factorization of large covariance matrix in the evaluation of the log-likelihood function, which makes the optimization time-prohibitive. For fast likelihood based inference, several approximate methods have been introduced in the literature, such as those based on hierarchical matrices techniques (Litvinenko et al., 2019), Whittle loglikelihood maximization (Whittle, 1954; Guinness et al., 2014) and step-wise maximum loglikelihood estimation (Li and Zhang, 2011; Apanasovich, Genton, and Sun, 2012). Here, we mainly focus on model construction instead of developing more sophisticated optimization techniques for large-scale spatial problems, which is still a challenging research area. Therefore, we choose a simple step-wise approach to estimate $\boldsymbol{\theta}^{\mathrm{a}}$ by two-step Gaussian likelihood maximization.

Let $\tilde{\mathbf{X}}=\left\{\mathbf{X}_{1}^{\mathrm{T}}, \ldots, \mathbf{X}_{p}^{\mathrm{T}}\right\}^{\mathrm{T}}$ be a realization from the $p$-variate random field $\mathbf{X}(\cdot)$ such that $\mathbf{X}_{i}=\left\{X_{i}\left(\mathbf{s}_{1}\right), \ldots, X_{i}\left(\mathbf{s}_{n}\right)\right\}^{\mathrm{T}}, i=1, \ldots, p$. Let $\boldsymbol{\Sigma}_{\text {full }}$ be the $n p \times n p$ covariance matrix for $\tilde{\mathbf{X}}$ where $\left\{\mathbf{C}_{q, r}^{\mathrm{a}}\left(\mathbf{s}_{i}-\mathbf{s}_{j}\right)\right\}_{i, j=1}^{n} \in \mathbb{R}^{n \times n}$ constitutes the $(q, r)$ th, $q, r=1, \ldots, p$, block entry of $\boldsymbol{\Sigma}_{\text {full }}$, and $\boldsymbol{\Sigma}_{\text {block }}$ be the $n p \times n p$ block diagonal covariance matrix ignoring the crosscovariances. Here, $\boldsymbol{\Sigma}_{\text {block }}$ depends only on $\boldsymbol{\theta}_{\mathrm{m}}$ whereas $\boldsymbol{\Sigma}_{\text {full }}$ depends on $\boldsymbol{\theta}^{\text {a }}$. We consider the composite loglikelihood $\ell^{\operatorname{comp}}\left(\boldsymbol{\theta}_{\mathrm{m}} \mid \tilde{\mathbf{X}}\right)=-\left(\log \operatorname{det} \boldsymbol{\Sigma}_{\text {block }}+\tilde{\mathbf{X}}^{\mathrm{T}} \boldsymbol{\Sigma}_{\text {block }}^{-1} \tilde{\mathbf{X}}+n p \log 2 \pi\right) / 2$ and the full loglikelihood $\ell^{\text {full }}\left(\boldsymbol{\theta}^{\mathrm{a}} \mid \tilde{\mathbf{X}}\right)=-\left(\log \operatorname{det} \boldsymbol{\Sigma}_{\text {full }}+\tilde{\mathbf{X}}^{\mathrm{T}} \boldsymbol{\Sigma}_{\text {full }}^{-1} \tilde{\mathbf{X}}+n p \log 2 \pi\right) / 2$ for optimization. We estimate the set of parameters $\boldsymbol{\theta}^{\mathrm{a}}$ as follows: (1) Obtain $\hat{\boldsymbol{\theta}}_{\mathrm{m}}=\arg \max _{\boldsymbol{\theta}_{m}} \ell^{\operatorname{comp}}\left(\boldsymbol{\theta}_{\mathrm{m}} \mid \tilde{\mathbf{X}}\right)$, (2) Obtain $\left(\hat{\boldsymbol{\theta}}_{\mathrm{c}}, \hat{\boldsymbol{\theta}}_{\mathrm{s}}\right)=\arg \max _{\left(\boldsymbol{\theta}_{\mathrm{c}}, \boldsymbol{\theta}_{\mathrm{s}}\right)} \ell^{\text {full }}\left(\boldsymbol{\theta}_{\mathrm{c}}, \boldsymbol{\theta}_{\mathrm{s}} \mid \tilde{\mathbf{X}}, \hat{\boldsymbol{\theta}}_{\mathrm{m}}\right)$ by using numerical maximization. Furthermore, we compute the numerically differentiated Hessian matrix in each of the two steps to obtain the approximate standard errors for $\hat{\boldsymbol{\theta}}_{\mathrm{m}}$ and $\left(\hat{\boldsymbol{\theta}}_{\mathrm{c}}, \hat{\boldsymbol{\theta}}_{\mathrm{s}}\right)$ in step one and step two, respectively.

For the trivariate case, we need to satisfy the sufficient condition of Theorem 2 during the estimation. Essentially, we need to satisfy the inequality of type $\sum_{i<j}^{p}\left|\beta_{i j}^{*}\right|^{2} \leq 1-2 \prod_{i<j}^{p}\left|\beta_{i j}^{*}\right|$, 
where $\beta_{i j}^{*}$ are some model specific functions of the elements in the set $\left\{\boldsymbol{\theta}_{\mathrm{m}}, \boldsymbol{\theta}_{\mathrm{c}}\right\}$. One way to satisfy the inequality is through re-parameterization of $\beta_{i j}^{*}$, and a valid re-parameterization is $\beta_{12}^{*}=(1-\delta) \sin \psi_{1} \cos \psi_{2} / \sqrt{2}, \beta_{13}^{*}=(1-\delta) \sin \psi_{1} \sin \psi_{2} / \sqrt{2}, \beta_{23}^{*}=(1-\delta) \cos \psi_{1} / \sqrt{2}$, where $0 \leq \delta \leq 1,0 \leq \psi_{1} \leq \pi$ and $0 \leq \psi_{2} \leq 2 \pi$

\section{Simulation Study}

\subsection{Simulation Setup}

We compare the goodness-of-fit and prediction performance of our model with Li and Zhang's asymmetric model and the corresponding symmetric model through multiple cases of simulation. We use the following notation: ${ }_{\mathrm{k}} \mathbf{C}^{\mathrm{a}}(\mathbf{h})$ denotes our proposed asymmetric model, ${ }_{\mathrm{k}} \mathbf{C}^{\mathrm{LZ}}(\mathbf{h})$ denotes Li and Zhang's asymmetric model and ${ }_{\mathrm{k}} \mathbf{C}(\mathbf{h})$ denotes the corresponding symmetric model. The subscript " $\mathrm{k}$ " denotes the class of underlying symmetric multivariate covariance models. Then, $\mathrm{k}=1$ denote the parsimonious multivariate Matérn model and $\mathrm{k}=2$ to denote the separable model with Matérn correlation function.

We simulate 100 realizations of a zero mean trivariate stationary Gaussian random field $\mathbf{X}(\mathbf{s})=\left\{X_{1}(\mathbf{s}), X_{2}(\mathbf{s}), X_{3}(\mathbf{s})\right\}^{\mathrm{T}}$ over $20 \times 20$ grid points in a spatial domain $[0,1]^{2}$, for six different cases of covariance structures. For Case 1, Cases 2-3 and Cases 4-5, we simulate $\mathbf{X}(\mathbf{s})$ with ${ }_{1} \mathbf{C}(\mathbf{h}),{ }_{1} \mathbf{C}^{\mathrm{a}}(\mathbf{h})$ and ${ }_{1} \mathbf{C}^{\mathrm{LZ}}(\mathbf{h})$, respectively, with the parameter settings as listed in Table 1. Lastly, for Case 6, we simulate $\mathbf{X}(\mathbf{s})$ from the following construction: $X_{i}(\mathbf{s})=W_{2 i-1}\left(\mathbf{s}-\tilde{\ell}_{i}\right)+W_{2 i}(\mathbf{s}), i=1,2,3$, where $\mathbf{W}(\mathbf{s})=\left\{W_{1}(\mathbf{s}), \ldots, W_{6}(\mathbf{s})\right\}^{\mathrm{T}}$ is a 6 -variate zero mean Gaussian random field with its covariance structure described by the parsimonious multivariate Matérn model with $a=7, \nu_{11}=\nu_{22}=2.75, \nu_{33}=\nu_{44}=1.5, \nu_{55}=\nu_{66}=$ $3, \rho_{12}=\rho_{15}=\rho_{16}=\rho_{24}=\rho_{34}=\rho_{35}=\rho_{36}=\rho_{56}=0, \rho_{13}=0.525, \rho_{14}=0.477, \rho_{23}=$ 0.095, $\rho_{25}=\rho_{26}=0.199, \rho_{45}=\rho_{46}=0.188$ and $\sigma_{i}=\sqrt{0.5}, i=1, \ldots, 6$. Here, the asymmetry is controlled by the parameters $\tilde{\boldsymbol{\ell}}_{1}=(0.17,0.17), \tilde{\boldsymbol{\ell}}_{2}=(0.09,0.09)$ and $\tilde{\boldsymbol{\ell}}_{3}=\mathbf{0}$. The 
cross-covariance functions for each case exhibit a distinct feature regarding its asymmetric behavior (see Figure 2), which we discuss in more detail in Section 3.2.

We fit the six candidate models ${ }_{k} \mathbf{C}^{\mathrm{a}}(\mathbf{h}),{ }_{\mathrm{k}} \mathbf{C}^{\mathrm{LZ}}(\mathbf{h})$ and ${ }_{\mathrm{k}} \mathbf{C}(\mathbf{h}), \mathrm{k}=1,2$, for each of the 100 realizations under each of the six cases. For the estimation of ${ }_{k} \mathbf{C}^{\mathrm{a}}(\mathbf{h}), \mathrm{k}=1,2$, we apply the two-step maximum likelihood estimation procedure discussed in Section 2.2. For the estimation of ${ }_{k} \mathbf{C}^{\mathrm{LZ}}(\mathbf{h})$ and ${ }_{\mathrm{k}} \mathbf{C}(\mathbf{h})(\mathrm{k}=1,2)$, we adapt the same two-step estimation procedure by removing the requirement of satisfying sufficient condition mentioned in Theorem 2 and redefining $\boldsymbol{\theta}_{s}$ according to the respective model specification. Additionally, we set $\boldsymbol{\ell}_{1}=\mathbf{0}$ to avoid identifiability issues in the estimation of ${ }_{k} \mathbf{C}^{\mathrm{LZ}}(\mathbf{h}), \mathrm{k}=1,2$, (Li and Zhang, 2011). We then assess the six candidate models in terms of the goodness-of-fit in each of the six cases by comparing the maximized loglikelihood ( $\log \mathrm{L})$ and the Akaike information criterion (AIC) associated with these estimated models. We also compare the six candidate models based on their prediction performance using the prediction scores, such as mean squared prediction error (MSPE) and mean logarithmic score (mLogS) (Gneiting and Raftery, 2007). A smaller value of these scores indicates better predictions. For computation of these scores, we perform a leave-one-out "pseudo cross-validation" study, similar to the one conducted in Genton and Kleiber (2015), where each time we predict one left out observation based on all other observations by using cokring methods (Cressie, 1993) with candidate models estimated from the full data.

\subsection{Simulation Results}

To give a broad summary of the goodness-of-fit, we report the average and standard error (SE) of LogL and AIC over 100 replicates in Table 2. For summarizing the prediction performances of all the candidate models, we report the percentage reduction of the averaged value $(\Delta . \mathrm{A})$ and standard error $(\Delta . \mathrm{SE})$ of MSPE and mLogS for each candidate models 
against ${ }_{1} \mathbf{C}(\mathbf{h})$ as the base case in Table 3 .

Based on the averaged values reported in Tables 2-3, we infer that there is a substantial improvement in terms of goodness-of-fit and spatial predictions by using the asymmetric models ${ }_{\mathrm{k}} \mathbf{C}^{\mathrm{a}}(\mathbf{h})$ and ${ }_{\mathrm{k}} \mathbf{C}^{\mathrm{LZ}}(\mathbf{h})$ over the symmetric models ${ }_{\mathrm{k}} \mathbf{C}(\mathbf{h}), \mathrm{k}=1,2$, in Cases $2-6$, whereas there is no such improvement in Case 1. This is to be expected because the underlying cross-covariance functions in Cases 2-6 exhibit asymmetry in at least one pair of variables (see Figures 2(d)-2(r)) whereas such asymmetry is absent in Case 1 (see Figures 2(a)-2(c)). Furthermore, the performance of models ${ }_{2} \mathbf{C}^{\mathrm{a}}(\mathbf{h}),{ }_{2} \mathbf{C}^{\mathrm{LZ}}(\mathbf{h})$ and ${ }_{2} \mathbf{C}(\mathbf{h})$ is always inferior to ${ }_{1} \mathbf{C}^{\mathrm{a}}(\mathbf{h}),{ }_{1} \mathbf{C}^{\mathrm{LZ}}(\mathbf{h})$ and ${ }_{1} \mathbf{C}(\mathbf{h})$, respectively, in all six cases because the simulated random fields exhibit distinct smoothness in different components (see Table 1) whereas the separable Matérn model allows only identical smoothness in all the process components. Additionally, the proposed models ${ }_{\mathrm{k}} \mathbf{C}^{\mathrm{a}}(\mathbf{h})$ remarkably outperforms ${ }_{\mathrm{k}} \mathbf{C}^{\mathrm{LZ}}(\mathbf{h})$ and ${ }_{\mathrm{k}} \mathbf{C}(\mathbf{h}), \mathrm{k}=1,2$, in Case 2, Case 3 and Case 6 .

The true underlying absolute coherence functions in Cases 1-4 satisfy the sufficient condition in Theorem 2, in which case, the models ${ }_{k} \mathbf{C}^{\mathrm{LZ}}(\mathbf{h})$ and ${ }_{\mathrm{k}} \mathbf{C}(\mathbf{h})$ can be considered as a particular case of ${ }_{\mathrm{k}} \mathbf{C}^{\mathrm{a}}(\mathbf{h}), \mathrm{k}=1,2$. Consequently, the candidate models ${ }_{1} \mathbf{C}^{\mathrm{a}}(\mathbf{h}),{ }_{1} \mathbf{C}^{\mathrm{LZ}}(\mathbf{h})$ and ${ }_{1} \mathbf{C}(\mathbf{h})$ provide qualitatively similar prediction scores in Case 1 , where ${ }_{1} \mathbf{C}(\mathbf{h})$ is the true model. However, in Case 1, the AIC for ${ }_{1} \mathbf{C}^{\mathrm{a}}(\mathbf{h})$ and ${ }_{1} \mathbf{C}^{\mathrm{LZ}}(\mathbf{h})$ is slightly higher than that of ${ }_{1} \mathbf{C}(\mathbf{h})$ because of the additional vector-valued asymmetry parameters, which could not lead to a significant increase in $\log L$.

In Case 2 and Case 3, the candidate model ${ }_{1} \mathbf{C}^{\mathrm{a}}(\mathbf{h})$ exceptionally outperforms all the other candidate models both in terms of goodness-of-fit and the prediction performance. Interestingly, the asymmetric models ${ }_{2} \mathbf{C}^{\mathrm{a}}(\mathbf{h})$ and ${ }_{2} \mathbf{C}^{\mathrm{LZ}}(\mathbf{h})$, which are based on the separable Matérn model, performs significantly better predictions in terms of MSPE than the relatively more flexible parsimonious multivariate Matérn based model ${ }_{1} \mathbf{C}(\mathbf{h})$ in Case 3 . The 
percentage reduction of MSPE for ${ }_{2} \mathbf{C}^{\mathrm{a}}(\mathbf{h})$ and ${ }_{2} \mathrm{C}^{\mathrm{LZ}}(\mathbf{h})$ are $10.9 \%$ and $7.0 \%$, respectively, thus exemplifying a case where modeling asymmetry is more crucial than the flexible choice of underlying covariance functions. Moreover, the model ${ }_{1} C^{a}(h)$ improves the prediction by nearly 1.7 and 1.2 times of the improvement achieved by using the model ${ }_{1} \mathbf{C}^{\mathrm{LZ}}(\mathbf{h})$ in terms of MSPE for Case 2 and Case 3, respectively. The poor performance of ${ }_{1} \mathbf{C}(\mathbf{h})$ and ${ }_{2} \mathbf{C}(\mathbf{h})$ in Cases 2-3 is obvious because they cannot capture the asymmetric spatial dependence between variables, which is inherent in the simulated random field. However, the inferior performance of ${ }_{1} \mathbf{C}^{\mathrm{LZ}}(\mathbf{h})$ relative to ${ }_{1} \mathbf{C}^{\mathrm{a}}(\mathbf{h})$ in Case 2 is attributed to the fact that the simulated trivariate random field exhibits asymmetric spatial dependence only between one pair of variables out of the three pairs of variables (see Figures $2(\mathrm{~d})-2(\mathrm{f}))$ and the model ${ }_{1} \mathbf{C}^{\mathrm{LZ}}(\mathbf{h})$ fails to describe such a covariance structure due to its restricted asymmetry specification in a trivariate case. Moreover, in Case 3, the simulated random field exhibits asymmetric spatial dependence in all three pairs of variables such that the extreme correlation occurs at the same lag $\mathbf{h}=(-0.15,-0.15)$ in all three pairs (see Figures $2(\mathrm{~g})-2(\mathrm{i})$ ). While such asymmetry is completely ignored by the symmetric models ${ }_{1} \mathbf{C}(\mathbf{h})$ and ${ }_{2} \mathbf{C}(\mathbf{h})$, it can be captured only partially by the asymmetric models ${ }_{1} \mathbf{C}^{\mathrm{LZ}}(\mathbf{h})$ and ${ }_{2} \mathrm{C}^{\mathrm{LZ}}(\mathbf{h})$. For instance, if ${ }_{\mathrm{k}} \mathrm{C}^{\mathrm{LZ}}(\mathrm{h}), \mathrm{k}=1,2$, perfectly captures the asymmetric spatial dependence of $X_{1}$ with $X_{2}$ and $X_{3}$, then the corresponding asymmetry parameters are $\boldsymbol{\ell}_{1}=\mathbf{0}, \boldsymbol{\ell}_{2}=(-0.15,-0.15)$ and $\ell_{3}=(-0.15,-0.15)$, and in that case the spatial dependence between $X_{2}$ and $X_{3}$ is forced to be symmetric because $\boldsymbol{\ell}_{2}-\boldsymbol{\ell}_{3}=\mathbf{0}$. Therefore, in Case 3 , the asymmetric models ${ }_{1} \mathbf{C}^{\mathrm{LZ}}(\mathbf{h})$ and ${ }_{2} \mathrm{C}^{\mathrm{LZ}}(\mathbf{h})$ can capture the asymmetric spatial dependence satisfactorily between only two pairs of variables out of the three pairs, which makes their goodness-of-fit and prediction performance inferior to the asymmetric models ${ }_{1} C^{\mathrm{a}}(\mathrm{h})$ and ${ }_{2} \mathrm{C}^{\mathrm{a}}(\mathrm{h})$, respectively.

In Cases $4-5,{ }_{1} \mathbf{C}^{\mathrm{LZ}}(\mathbf{h})$ is the true covariance model (see Figures $2(\mathrm{j})-2(\mathrm{o})$ ) for the simulated random fields and consequently the candidate model ${ }_{1} \mathrm{C}^{\mathrm{LZ}}(\mathbf{h})$ produces the lowest 
AIC. While the asymmetry in cross-covariances are identical for both Case 4 and Case 5, the strength of true cross-covariance is much stronger in the latter. The true absolute coherence function in Case 4 satisfies the inequality of Theorems 1-2, whereas Case 5 exhibit the most extreme violation of the inequality. Accordingly, the difference in performance of ${ }_{1} \mathbf{C}^{\mathrm{a}}(\mathbf{h})$ and ${ }_{1} \mathbf{C}^{\mathrm{LZ}}(\mathbf{h})$ is noticeably severe in Case 5 , whereas it is negligible in Case 4. Both of the asymmetric models ${ }_{1} \mathbf{C}^{\mathrm{LZ}}(\mathbf{h})$ and ${ }_{1} \mathbf{C}^{\mathrm{a}}(\mathbf{h})$ demonstrate equally good prediction performance in Case 4. However, for Case $5,{ }_{1} \mathbf{C}^{\mathrm{LZ}}(\mathbf{h})$ improves the prediction by nearly 4 times of the improvement achieved by using the model ${ }_{1} \mathbf{C}^{\mathrm{a}}(\mathbf{h})$ in terms of MSPE. The suboptimal performance of ${ }_{1} \mathbf{C}^{\mathrm{a}}(\mathbf{h})$ relative to ${ }_{1} \mathbf{C}^{\mathrm{LZ}}(\mathbf{h})$ in Case 5 is attributed to its truncated parameter space for the absolute coherence function which could not capture concurrently high coherence functions in the data.

In Case 6, all the six candidate models are misspecified for modeling the true crosscovariance functions underlying the simulated random fields. This misspecification is primarily due to the fact that the true cross-covariances for $\mathbf{X}(\mathbf{s})$ are not of the Matérn type, as the true cross-covariances are the sum of symmetric and asymmetric Matérn covariance functions. Moreover, unlike all the other cases, here the cross-covariance between $X_{1}$ and $X_{2}$ does not exhibit the isotropic decay of covariance from the centre of the covariance structure (see Figure 2(p)). However, the models ${ }_{1} \mathbf{C}^{\mathrm{a}}(\mathbf{h}),{ }_{1} \mathbf{C}^{\mathrm{LZ}}(\mathbf{h})$ and ${ }_{1} \mathbf{C}(\mathbf{h})$ can recover the true marginal covariance functions because the true marginal covariance functions underlying the simulated random fields belong to the Matérn class with distinct smoothnesses in each component for the parameter setting mentioned in Section 3.1. Among all the six misspecified candidate models, the asymmetric model ${ }_{1} \mathbf{C}^{\mathrm{a}}(\mathbf{h})$ demonstrates a significantly superior fit to the data relative to all the other candidate models. The AIC for the asymmetric model ${ }_{1} \mathbf{C}^{\mathrm{a}}(\mathbf{h})$ is reasonably lower than that of the asymmetric model ${ }_{1} \mathbf{C}^{\mathrm{LZ}}(\mathbf{h})$ and the symmetric model ${ }_{1} \mathbf{C}(\mathbf{h})$. The asymmetric model ${ }_{1} \mathbf{C}^{\mathrm{a}}(\mathbf{h})$ also produces the best prediction 
scores among all the other candidate models. The superior performance of the asymmetric model ${ }_{1} \mathbf{C}^{\mathrm{a}}(\mathbf{h})$ whereas no significant modeling improvement by using the asymmetric model ${ }_{1} \mathbf{C}^{\mathrm{LZ}}(\mathbf{h})$ against ${ }_{1} \mathbf{C}(\mathbf{h})$ is due to the inherent asymmetric spatial dependence between $X_{1}$ and $X_{2}$ in the simulated random fields. While the symmetric model ${ }_{1} \mathbf{C}(\mathbf{h})$ completely ignores this asymmetry, the asymmetric model ${ }_{1} \mathbf{C}^{\mathrm{LZ}}(\mathbf{h})$ is also not beneficial here because, as already mentioned, it fails to describe asymmetric spatial dependence if it is present only in one out of three pairs of variables. Although the asymmetric model ${ }_{1} \mathbf{C}^{\mathrm{a}}(\mathbf{h})$ cannot recover the true underlying cross-covariance, its flexible specification for modeling asymmetry allows to recover it quite closely and this makes it the best candidate model in Case 6 .

\section{Applications to $\mathrm{PM}_{2.5}$, Wind Speed and Relative $\mathrm{Hu}-$ midity Data}

Particulate matter $\left(\mathrm{PM}_{2.5}\right)$ represents the concentration of fine particulate matter suspended in the air whose diameter is less than $2.5 \mu \mathrm{m}$, and its exposure in high concentrations is well known to have hazardous effects on human health (Samoli et al., 2008; Chang, Reich, and Miranda, 2011). Concentrations of $\mathrm{PM}_{2.5}$ in a region are strongly influenced by meteorological conditions such as cloud cover, precipitation, relative humidity, temperature, wind speed, etc., (Tai, Mickley, and Jacob, 2010). In this work, we apply our modeling approach to study the dynamics of $\mathrm{PM}_{2.5}$ and two meteorological variables, namely wind speed and relative humidity, which in general show negative and positive correlations with $\mathrm{PM}_{2.5}$, respectively (Dawson, Adams, and Pandis, 2007; Jacob and Winner, 2009). We explore the marginal and potentially asymmetric cross-spatial dependence in these variables using our proposed model. We also compare the application of our model to this dataset with Li and Zhang's model and the corresponding symmetric model on the basis of goodness-of-fit and spatial predictions. 
We consider the spatial domain of interest to be the north-eastern climatic region of the United States defined by the National Climatic Data Center (Karl and Koss, 1984). The data for $\mathrm{PM}_{2.5}$ that we consider is an output from the Community Multiscale Air Quality Modeling System (CMAQ) developed by the Environmental Protection Agency (EPA) (CMAQ, https://www.epa.gov/cmaq), which provides the daily average values. For the meteorological variables, we obtain the data for wind speed at 1000mb (WS) and relative humidity at $2 \mathrm{~m}(\mathrm{RH})$ from the North American Regional Reanalysis (NARR, https://www.esrl.noaa.gov/psd), which provides the monthly mean values of various meteorological variables. From these raw datasets, we prepare a co-located trivariate dataset for monthly mean $\mathrm{PM}_{2.5}$ /WS/RH for July, 2012, over 481 observed locations shown in Figure 3.

While WS exhibits an approximately Gaussian distribution already, we log transform $\mathrm{PM}_{2.5}$ and $\mathrm{RH}$ to make them approximately Gaussian. We begin the analysis by removing the spatial trend in the directions of longitude and latitude by using the linear regression in all the three variables, which are $\log \mathrm{PM}_{2.5}$, WS and $\log \mathrm{RH}$. The detrended variables are then standardized to make it suitable for modeling as a zero mean trivariate Gaussian random field. Let us assume that $\mathbf{Y}(\mathbf{s})=\left\{Y_{1}(\mathbf{s}), Y_{2}(\mathbf{s}), Y_{3}(\mathbf{s})\right\}^{\mathrm{T}}$ is a zero mean trivariate Gaussian random field where $Y_{1}, Y_{2}$ and $Y_{3}$ represent the detrended and standardized $\log \mathrm{PM}_{2.5}$, WS and $\log \mathrm{RH}$, respectively.

We consider the flexible multivariate Matérn model to be suitable for modeling the trivariate field $\mathbf{Y}$, since it allows spatial scale and smoothness to vary in process components. However, to reduce the burden on optimization due to overwhelming number of parameters, we constraint the cross-smoothness parameter $\nu_{i j}$ and the cross-spatial scale parameter $a_{i j}$ as: (1) $\nu_{i j}=\left(\nu_{i i}+\nu_{j j}\right) / 2$ and $(2) a_{i j}^{2}=\left(a_{i i}^{2}+a_{j j}^{2}\right) / 2$. Furthermore, we re-parameterize $a_{i j}=2 \sqrt{\nu_{i j}} a, a>0$, to satisfy constraint (2). Let $\mathrm{k}=3$ denote the flexible multivariate Matérn model in the notation defined in Section 3.1, then we fit three candidate models 
${ }_{3} \mathbf{C}^{\mathrm{a}}(\mathbf{h}),{ }_{3} \mathbf{C}^{\mathrm{LZ}}(\mathbf{h})$ and ${ }_{3} \mathbf{C}(\mathbf{h})$, augmented with nugget variances $\delta_{i}>0, \quad i=1,2,3$, on the observations of the trivariate field $\mathbf{Y}$ using the two-step maximum likelihood estimation, as executed in Section 3. For satisfactory estimation of asymmetric cross-covariance functions in ${ }_{3} \mathbf{C}^{\mathrm{a}}(\mathbf{h})$ and ${ }_{3} \mathbf{C}^{\mathrm{LZ}}(\mathbf{h})$, the choice of initial values for the asymmetry parameters is crucial in the numerical maximization. In order to find reasonably good initial values of asymmetry parameters $\boldsymbol{\ell}_{i j}, i=1,2,3$, for the estimation of ${ }_{3} \mathbf{C}^{\mathrm{a}}(\mathbf{h})$, we perform a grid search of parameter values by considering two variables at a time. As noted already in Section 2.1, the two asymmetric models ${ }_{3} \mathbf{C}^{\mathrm{a}}(\mathbf{h})$ and ${ }_{3} \mathbf{C}^{\mathrm{LZ}}(\mathbf{h})$ are equivalent in the case of the bivariate data. For the given marginal parameter estimates, we evaluate the full loglikelihood of the bivariate model ${ }_{3} \mathbf{C}^{\mathrm{a}}(\mathbf{h})$ based on the bivariate data $\mathbf{Y}_{i j}(\mathbf{s})=\left\{Y_{i}(\mathbf{s}), Y_{j}(\mathbf{s})\right\}^{\mathrm{T}}$, over a grid of values of parameters $\beta_{i j}$ and $\boldsymbol{\ell}_{i j}$. The range for $\beta_{i j}$ in the grid is set to be $[-1,1]$, whereas the range for $\boldsymbol{\ell}_{i j}$ is set to be the minimum and maximum possible spatial lags in the spatial domain, which in our case is $(-1081.87 \mathrm{~km}, 1081.87 \mathrm{~km}) \times(-1070.295 \mathrm{~km}, 1070.295 \mathrm{~km})$. The maximizer of the full bivariate loglikelihood from the grid search is then supplied as initial values in numerical maximization of the full bivariate loglikelihood to obtain the estimates ${ }_{b f} \hat{\ell_{i j}}$ and ${ }_{b f} \hat{\beta_{i j}}$ based on the bivariate fits. These estimates ${ }_{b f} \hat{\ell_{12}},{ }_{b f} \hat{\ell_{13}}$ and ${ }_{b f} \hat{\ell_{23}}$ are then considered as the initial values for $\ell_{12}, \ell_{13}$ and $\ell_{23}$, respectively, in the numerical maximization of full loglikelihood of the model ${ }_{3} \mathbf{C}^{\mathrm{a}}(\mathbf{h})$ for the trivariate field $\mathbf{Y}$. The estimates ${ }_{b f} \hat{\ell_{12}},{ }_{b f} \hat{\ell_{13}}$ and ${ }_{b f} \hat{\ell_{23}}$ are also used for finding initial values in the estimation of ${ }_{3} \mathbf{C}^{\mathrm{LZ}}(\mathbf{h})$. We try three sets of initial values $\left({ }_{b f} \hat{\ell_{12}},{ }_{b f} \hat{\ell_{13}}\right),\left({ }_{b f} \hat{\ell_{12}},{ }_{b f} \hat{\ell_{12}}+{ }_{b f} \hat{\ell_{23}}\right)$ and $\left({ }_{b f} \hat{\ell_{13}}-{ }_{b f} \hat{\ell_{23}},{ }_{b f} \hat{\ell_{13}}\right)$ for the parameters $\left(\boldsymbol{\ell}_{2}, \boldsymbol{\ell}_{3}\right)$ in the numerical maximization of full loglikelihood based on the model ${ }_{3} \mathbf{C}^{\mathrm{LZ}}(\mathbf{h})$.

Table 4 reports the estimated parameters with their standard errors and time to fit the three candidate models considered. The timings are based on computations in $\mathrm{R}$ ( $\mathrm{R}$ Core Team, 2017) on a MacBook Pro laptop with 2.5 GHz Intel Core i7 processor and 16 GB of $1600 \mathrm{MHz}$ DDR3 RAM. Note that the reported computational time is the sum of time taken 
to optimize the loglikelihood function in step one and step two, and does not include the computational time to obtain ${ }_{b f} \hat{\beta_{i j}}$, ${ }_{b f} \hat{\ell_{i j}}, 1 \leq i \neq j \leq 3$, through grid search and bivariate estimations. The computational time for finding good initial values for both the asymmetric models in terms of ${ }_{b f} \hat{\beta_{i j}},{ }_{b f} \hat{\ell_{i j}}, 1 \leq i \neq j \leq 3$, in our is case 15516.9 seconds.

Based on the three fitted candidate models, we compute the MSPE and mLogS by performing the leave-one-out pseudo cross-validation study. The goodness-of-fit summary and prediction scores are reported in Table 5. Both the asymmetric models ${ }_{3} \mathbf{C}^{\mathrm{a}}(\mathbf{h})$ and ${ }_{3} \mathbf{C}^{\mathrm{LZ}}(\mathbf{h})$ lead to a significant improvement in the goodness-of-fit and spatial predictions compared to the symmetric model ${ }_{3} \mathbf{C}(\mathbf{h})$. Among the two asymmetric models, the proposed model ${ }_{3} \mathrm{C}^{\mathrm{a}}(\mathrm{h})$ slightly outperforms Li and Zhang's model ${ }_{3} \mathrm{C}^{\mathrm{LZ}}(\mathrm{h})$ both in terms of AIC and prediction scores. The percentage reduction of (MSPE, mLogS) by using ${ }_{3} \mathbf{C}^{\mathrm{a}}(\mathbf{h})$ against ${ }_{3} \mathbf{C}(\mathbf{h})$ is $(2.88 \%, 1.61 \%)$, which is higher than that of ${ }_{3} \mathrm{C}^{\mathrm{LZ}}(\mathbf{h})$, which leads to the percentage reduction of $(1.64 \%, 0.75 \%)$. We plot the estimated asymmetric cross-correlation functions for models ${ }_{3} \mathbf{C}^{\mathrm{a}}(\mathbf{h})$ and ${ }_{3} \mathbf{C}^{\mathrm{LZ}}(\mathbf{h})$ in Figures $4(\mathrm{a})-4(\mathrm{c})$ and Figures 4(e)-4(g), respectively. Both the asymmetric models ${ }_{3} \mathrm{C}^{\mathrm{a}}(\mathbf{h})$ and ${ }_{3} \mathrm{C}^{\mathrm{LZ}}(\mathbf{h})$ estimate nearly identical asymmetric cross-correlation functions for the two pair of variables $\left(Y_{1}, Y_{2}\right)$ and $\left(Y_{1}, Y_{3}\right)$; however, the estimates differ significantly for the third pair of variables $\left(Y_{2}, Y_{3}\right)$. This disparity in the asymmetric behavior for the pair $\left(Y_{2}, Y_{3}\right)$ is due to the fact that the model ${ }_{3} \mathbf{C}^{\mathrm{LZ}}(\mathbf{h})$ can independently specify asymmetry parameters for any two pairs of variables, whereas ${ }_{3} \mathbf{C}^{\mathrm{a}}(\mathbf{h})$ independently specifies asymmetry parameters for all three pairs of variables.

Additionally, we can also compare the two models ${ }_{3} \mathbf{C}^{\mathrm{a}}(\mathbf{h})$ and ${ }_{3} \mathrm{C}^{\mathrm{LZ}}(\mathbf{h})$ by the virtue of consistency of the estimated cross-correlation functions when moving from the bivariate case to the trivariate case. For bivariate data, the two models ${ }_{3} \mathbf{C}^{\mathrm{a}}(\mathbf{h})$ and ${ }_{3} \mathrm{C}^{\mathrm{LZ}}(\mathbf{h})$ are equivalent. Figure 4(d) (or equivalently Figure 4(h)) shows the estimated cross-correlation function of $Y_{2}$ and $Y_{3}$ from the bivariate fit of the model ${ }_{3} \mathrm{C}^{\mathrm{a}}(\mathbf{h})$ (or equivalently ${ }_{3} \mathrm{C}^{\mathrm{LZ}}(\mathbf{h})$ ). 
Its comparison with the completely different cross-correlation function estimate of $Y_{2}$ and $Y_{3}$ from the trivariate fit of the model ${ }_{3} \mathbf{C}^{\mathrm{LZ}}(\mathbf{h})$ (shown in Figure $4(\mathrm{~g})$ ) clearly reveals the inconsistency of the model ${ }_{3} \mathbf{C}^{\mathrm{LZ}}(\mathbf{h})$, when the number of variables are increased from $p=2$ to $p=3$. While the bivariate fit of the model ${ }_{3} \mathbf{C}^{\mathrm{LZ}}(\mathbf{h})$ shows significantly positive asymmetric cross-correlations between $Y_{2}$ and $Y_{3}$, its trivariate fit exhibits near independence of $Y_{2}$ and $Y_{3}$. On the contrary, the cross-correlation of $Y_{2}$ and $Y_{3}$ from the trivariate fit of the model ${ }_{3} \mathbf{C}^{\mathrm{a}}(\mathbf{h})$ (shown in Figure 4(c)) shows close consistency with its bivariate fit (shown in Figure $4(\mathrm{~d}))$. Both the bivariate and trivariate fits of the model ${ }_{3} \mathbf{C}^{\mathrm{a}}(\mathbf{h})$ show similar asymmetric cross-correlation between $Y_{2}$ and $Y_{3}$ with only a slight difference in the scale of correlation. This difference is due to the constraints on the absolute coherence function (see Theorem 2) that slightly scales down the cross-correlations in the trivariate case to retain the validity of the corresponding trivariate cross-covariance model.

The prediction scores, AIC and consistency comparison suggests that the proposed candidate model ${ }_{3} \mathbf{C}^{\mathrm{a}}(\mathbf{h})$ is the best among the three candidate models to jointly explain the asymmetric cross-correlations of $\mathbf{Y}$. Based on the estimated trivariate model ${ }_{3} \mathbf{C}^{\mathrm{a}}(\mathbf{h})$, we infer that the variable $Y_{1}$ exhibits nearly symmetric spatial dependence with $Y_{2}$, whereas $Y_{3}$ shows strong asymmetric spatial dependence with both $Y_{1}$ and $Y_{2}$. The asymmetry between $Y_{2}$ and $Y_{3}$ exists in an approximately east-west direction, suggesting the effect of $Y_{2}$ is delayed on $Y_{3}$ in the eastern direction, such that the effect is maximum at a spatial lag of $\mathbf{h}=\hat{\mathbf{\ell}_{23}}=(793.38 \mathrm{Km}, 174.18 \mathrm{Km})$. On the other hand, the asymmetry between $Y_{1}$ and $Y_{3}$ exists in an approximately (north-east)-(south-west) direction. The effect of $Y_{1}$ is maximum with $Y_{3}$ at a spatial lag of $\mathbf{h}=\hat{\ell_{13}}=(-210.58 \mathrm{Km},-356.77 \mathrm{Km})$. Interestingly, the Pearson's correlation coefficient for the observed pairs of variables $\left(Y_{1}, Y_{2}\right),\left(Y_{1}, Y_{3}\right)$ and $\left(Y_{2}, Y_{3}\right)$ are $-0.11,-0.11$ and 0.14 , respectively, suggesting weaker cross-correlations. However, there exists a much stronger asymmetric spatial cross-correlations among these three 
variables, which have been revealed by the proposed asymmetric model in the joint modeling of all the variables.

\section{Discussion}

In this article, we have introduced an approach to impart flexible asymmetries in crosscovariances of stationary multivariate covariance functions. For the bivariate case $(p=2)$, the proposed model exactly coincides with an existing class of asymmetric model; however, for $p>2$ the proposed model offers a great deal of flexibility in specifying the asymmetry. We have presented multiple cases of simulation studies and a data application to demonstrate the improvement in goodness-of-fit and spatial predictions that can be achieved by using our proposed model against the corresponding symmetric and an existing class of asymmetric model for $p=3$. Additionally, we have also shown that the proposed model exhibits consistency of asymmetry controlling parameters when we add more variables, whereas the Li and Zhang model lacks such a desirable consistency feature.

The proposed approach puts constraints on the absolute coherence functions which restricts the strength of extreme cross-correlations. Consequently, as demontrated in Case 5 of the simulation study in Section 3, the proposed model underperforms when the true absolute coherence functions lie outside the truncated parameter space shown in Figure 1. Therefore, sub-optimal performance is expected if the absolute coherence function estimated using the proposed approach lie very close to the boundary of the truncated parameter space, as this hints towards the stronger underlying absolute coherence functions which the proposed method failed to capture due to the constraint in Theorems 2.

We have given sufficient validity conditions only for the case $p \leq 3$ (in Theorem 2), the generalization of the validity conditions for any specific higher value of $p$ is a possible future direction of research. Other potential future extensions include generalization of the 
proposed model for the non-stationary modeling framework. One possible direction for this non-trivial extension would require the asymmetry controlling vector-valued parameters $\boldsymbol{\ell}_{i j}$ to be spatially varying. Incorporating the proposed asymmetric model for a multivariate spatio-temporal modeling framework is also a plausible future prospect.

\section{References}

Apanasovich, T. V. and Genton, M. G. (2010). Cross-covariance functions for multivariate random fields based on latent dimensions. Biometrika 97, 15-30.

Apanasovich, T. V., Genton, M. G., and Sun, Y. (2012). A valid Matérn class of crosscovariance functions for multivariate random fields with any number of components. Journal of the American Statistical Association 107, 180-193.

Brown, P. J., Le, N. D., and Zidek, J. V. (1994). Multivariate spatial interpolation and exposure to air pollutants. Canadian Journal of Statistics 22, 489-509.

Chang, H. H., Reich, B. J., and Miranda, M. L. (2011). Time-to-event analysis of fine particle air pollution and preterm birth: Results from north carolina, 2001-2005. American Journal of Epidemiology 175, 91-98.

Christensen, W. and Amemiya, Y. (2001). Generalized shifted-factor analysis method for multivariate geo-referenced data. Mathematical Geology 33, 801-824.

Cramér, H. (1940). On the theory of stationary random processes. Annals of Mathematics 41, 215-230.

Cressie, N. (1993). Statistics for Spatial Data. Wiley, New york.

Cressie, N. and Zammit-Mangion, A. (2016). Multivariate spatial covariance models: a conditional approach. Biometrika 103, 915-935. 
Dawson, J. P., Adams, P. J., and Pandis, S. N. (2007). Sensitivity of $\mathrm{PM}_{2.5}$ to climate in the Eastern US: a modeling case study. Atmospheric Chemistry and Physics 7, 4295-4309.

Gaspari, G. and Cohn, S. E. (1999). Construction of correlation functions in two and three dimensions. Quarterly Journal of the Royal Meteorological Society 125, 723-757.

Gaspari, G., Cohn, S. E., Guo, J., and Pawson, S. (2006). Construction and application of covariance functions with variable length-fields. Quarterly Journal of the Royal Meteorological Society 132, 1815-1838.

Gelfand, A., Diggle, P., Guttorp, P., and Fuentes, M. (2010). Handbook of Spatial Statistics. Chapman \& Hall/CRC Handbooks of Modern Statistical Methods. CRC Press.

Genton, M. G. and Kleiber, W. (2015). Cross-covariance functions for multivariate geostatistics. Statistical Science 30, 147-163.

Gneiting, T., Kleiber, W., and Schlather, M. (2010). Matérn cross-covariance functions for multivariate random fields. Journal of the American Statistical Association 105, 11671177.

Gneiting, T. and Raftery, A. E. (2007). Strictly proper scoring rules, prediction, and estimation. Journal of the American Statistical Association 102, 359-378.

Goulard, M. and Voltz, M. (1992). Linear coregionalization model: Tools for estimation and choice of cross-variogram matrix. Mathematical Geology 24, 269-286.

Guinness, J., Fuentes, M., Hesterberg, D., and Polizzotto, M. (2014). Multivariate spatial modeling of conditional dependence in microscale soil elemental composition data. Spatial Statistics 9, 93 - 108. Revealing Intricacies in Spatial and Spatio-Temporal Data: Papers from the Spatial Statistics 2013 Conference. 
Helterbrand, J. D. and Cressie, N. (1994). Universal cokriging under intrinsic coregionalization. Mathematical Geology 26, 205-226.

Horn, R. A. and Johnson, C. R. (2013). Matrix analysis. Cambridge University Press, Cambridge, 2nd edition.

Jacob, D. J. and Winner, D. A. (2009). Effect of climate change on air quality. Atmospheric Environment 43, 51 - 63 .

Karl, T. R. and Koss, W. J. (1984). Regional and national monthly, seasonal, and annual temperature weighted by area, 1895-1983. National Climatic Data Center, Asheville, N.C.

Kleiber, W. (2017). Coherence for multivariate random fields. Statistica Sinica 27, 16751697.

Li, B., Genton, M., and Sherman, M. (2008). Testing the covariance structure of multivariate random fields. Biometrika 95, 813-829.

Li, B. and Zhang, H. (2011). An approach to modeling asymmetric multivariate spatial covariance structures. Journal of Multivariate Analysis 102, 1445 - 1453.

Litvinenko, A., Kriemann, R., Genton, M. G., Sun, Y., and Keyes, D. E. (2019). Hlibcov: Parallel hierarchical matrix approximation of large covariance matrices and likelihoods with applications in parameter identification. MethodsX.

Majumdar, A. and Gelfand, A. E. (2007). Multivariate spatial modeling for geostatistical data using convolved covariance functions. Mathematical Geology 39, 225-245.

Mardia, K. V. and Goodall, C. R. (1993). Spatial-temporal analysis of multivariate environmental monitoring data. In Multivariate Environmental Statistics. North-Holland Series in Statistics and Probability, volume 6, pages 347-386. North-Holland, Amsterdam. 
Priestley, M. B. (1981). Spectral analysis and time series, volume 1. Academic press London.

R Core Team (2017). R: A Language and Environment for Statistical Computing. R Foundation for Statistical Computing, Vienna, Austria.

Royle, J. A. and Berliner, L. M. (1999). A hierarchical approach to multivariate spatial modeling and prediction. Journal of Agricultural, Biological, and Environmental Statistics 4, 29-56.

Sain, S. and Cressie, N. (2007). A spatial model for multivariate lattice data. Journal of Econometrics 140, 226-259.

Sain, S. R., Furrer, R., and Cressie, N. (2011). A spatial analysis of multivariate output from regional climate models. The Annals of Applied Statistics 5, 150-175.

Samoli, E., Peng, R., Ramsay, T., Pipikou, M., Touloumi, G., Dominici, F., Burnett, R., Cohen, A., Krewski, D., Samet, J., and Katsouyanni, K. (2008). Acute effects of ambient particulate matter on mortality in Europe and North America: Results from the APHENA study. Environmental health perspectives 116, 1480-1486.

Schmidt, A. M. and Gelfand, A. E. (2003). A bayesian coregionalization approach for multivariate pollutant data. Journal of Geophysical Research: Atmospheres 108,.

Tai, A. P., Mickley, L. J., and Jacob, D. J. (2010). Correlations between fine particulate matter $\left(\mathrm{PM}_{2.5}\right)$ and meteorological variables in the United States: Implications for the sensitivity of $\mathrm{PM}_{2.5}$ to climate change. Atmospheric Environment 44, 3976 - 3984.

Ver Hoef, J. M. and Barry, R. P. (1998). Constructing and fitting models for cokriging and multivariable spatial prediction. Journal of Statistical Planning and Inference 69, $275-294$. 
Ver Hoef, J. M. and Cressie, N. (1993). Multivariable spatial prediction. Mathematical Geology 25, 219-240.

Ver Hoef, J. M., Cressie, N., and Barry, R. P. (2004). Flexible spatial models for kriging and cokriging using moving averages and the fast Fourier transform (FFT). Journal of Computational and Graphical Statistics 13, 265-282.

Wackernagel, H. (2003). Multivariate geostatistics: An Introduction with Applications. Berlin: Springer, 3rd edition.

Whittle, P. (1954). On stationary processes in the plane. Biometrika 41, 434-449.

Zhang, H. (2007). Maximum-likelihood estimation for multivariate spatial linear coregionalization models. Environmetrics 18, 125-139. 


\section{$6 \quad$ Figures and Tables}

Table 1: Parameter settings for simulation of $\mathbf{X}(\mathbf{s})$ in (a) Case 1 with ${ }_{1} \mathbf{C}(\mathbf{h})$, (b) Cases $2-3$ with ${ }_{1} \mathbf{C}^{\mathrm{a}}(\mathbf{h})$, and (c) Cases $4-5$ with ${ }_{1} \mathbf{C}^{\mathrm{LZ}}(\mathbf{h})$. The value of marginal standard deviations $\sigma_{i}$ for $X_{i}(\mathbf{s}), i=1,2,3$, is set to be equal to 1 , i.e, $\sigma_{i}=1, i=1,2,3$, in Cases $1-5$.

\begin{tabular}{ccccccccccc}
\hline \hline Case & \multicolumn{1}{c}{$\begin{array}{c}\text { Parsimonious multivariate } \\
\text { Matérn parameters }\end{array}$} & \multicolumn{5}{c}{ Asymmetry parameters } \\
& \multicolumn{1}{c}{$\nu_{11}$} & $\nu_{22}$ & $\nu_{33}$ & $\beta_{12}$ & $\beta_{13}$ & $\beta_{23}$ & - & - & - \\
\hline 1 & 7.5 & 1 & 2 & 3 & 0.65 & 0.39 & 0.45 & - & - & - \\
\hline & & & & & & & & $\boldsymbol{\ell}_{12}$ & $\boldsymbol{\ell}_{13}$ & $\boldsymbol{\ell}_{23}$ \\
\hline 2 & 7.5 & 1 & 2 & 3 & 0.65 & 0.39 & 0.45 & $(-0.15,-0.15)$ & $(0.00,0.00)$ & $(0.00,0.00)$ \\
\hline 3 & 7.5 & 1 & 2 & 3 & 0.65 & 0.39 & 0.45 & $(-0.15,-0.15)$ & $(-0.15,-0.15)$ & $(-0.15,-0.15)$ \\
\hline & & & & & & & & $\boldsymbol{\ell}_{1}$ & $\boldsymbol{\ell}_{2}$ & $\boldsymbol{\ell}_{3}$ \\
\hline 4 & 7.5 & 1 & 2 & 3 & 0.65 & 0.39 & 0.45 & $(0.00,0.00)$ & $(0.40,0.60)$ & $(0.30,0.40)$ \\
\hline 5 & 7.5 & 1 & 2 & 3 & 1.00 & 1.00 & 1.00 & $(0.00,0.00)$ & $(0.40,0.60)$ & $(0.30,0.40)$ \\
\hline
\end{tabular}




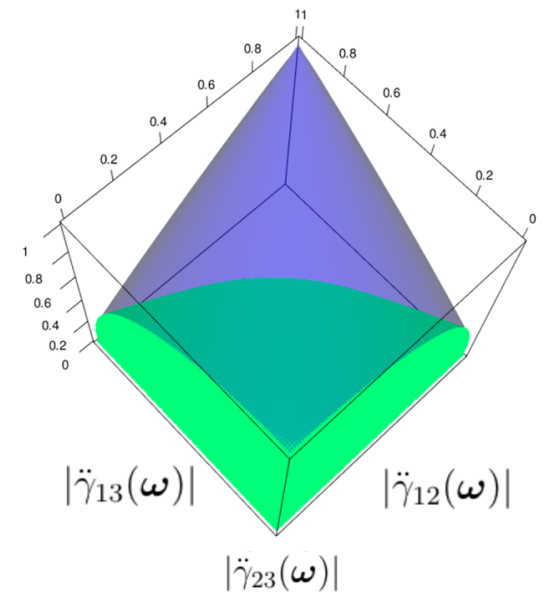

$\left|\ddot{\gamma}_{23}(\boldsymbol{\omega})\right|$

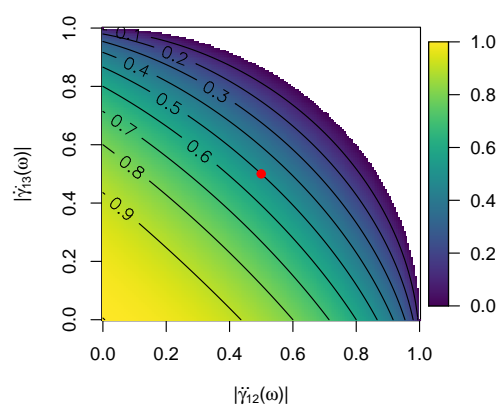

(b)

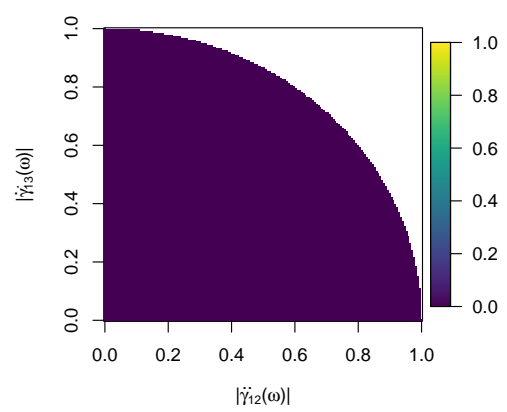

(e)

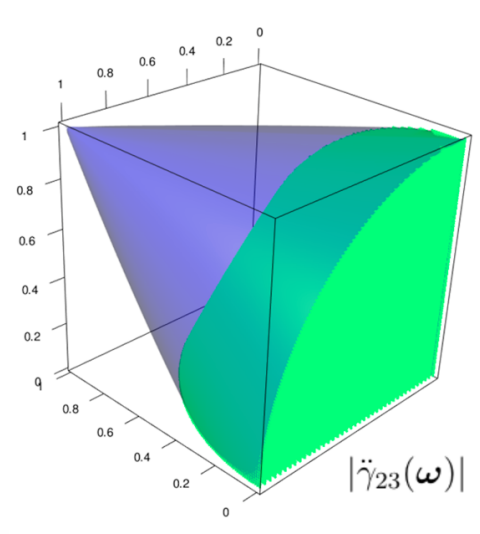

(a)

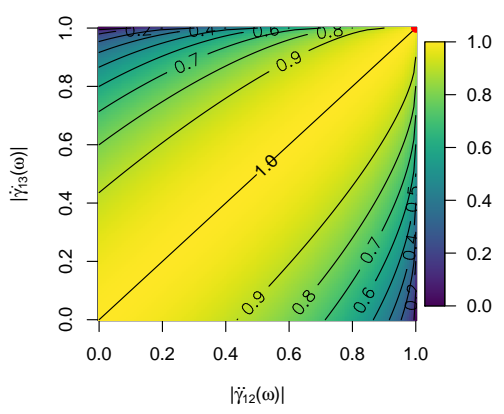

(c)

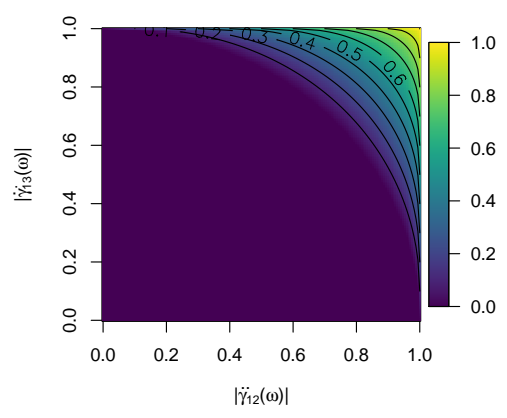

(f)
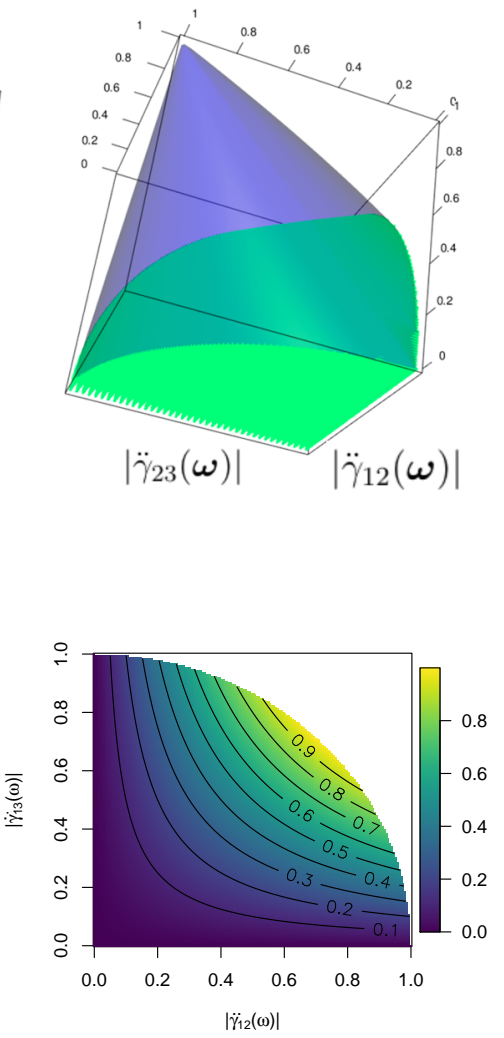

(d)

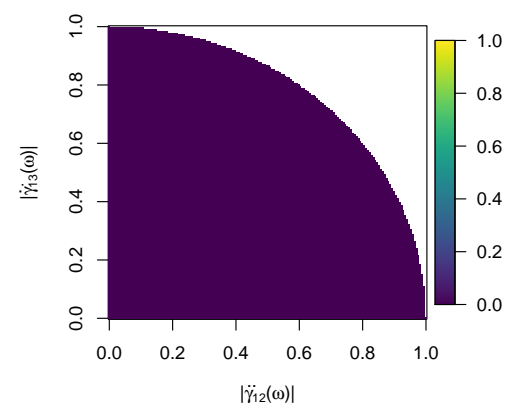

(g)

Figure 1: (a) Visualization of the parameter space of the absolute coherence functions $\left|\ddot{\gamma}_{i j}(\boldsymbol{\omega})\right|, i \neq j=1, \ldots, 3$, under the sufficient validity conditions for our proposed asymmetric model (green) and its symmetric counterpart (blue). Heatmap and contour lines for the upper bound of $\left|\ddot{\gamma}_{23}(\boldsymbol{\omega})\right|$ over different values of $\left(\left|\ddot{\gamma}_{12}(\boldsymbol{\omega})\right|,\left|\ddot{\gamma}_{13}(\boldsymbol{\omega})\right|\right)$ under the (b) proposed asymmetric model and (c) the corresponding symmetric model. (d) The reduction in the upper bounds, i.e., (c)-(b). Heatmap and contour lines for the lower bound of $\left|\ddot{\gamma}_{23}(\boldsymbol{\omega})\right|$ over different values of $\left(\left|\ddot{\gamma}_{12}(\boldsymbol{\omega})\right|,\left|\ddot{\gamma}_{13}(\boldsymbol{\omega})\right|\right)$ under the (e) proposed asymmetric model and (f) the corresponding symmetric model. (g) The reduction in the lower bounds, i.e., (e)-(f). White spaces in (b) and (e) shows the space where the inequality in Theorem 1 and Theorem 2 does not satisfy. Red point at $(0.5,0.5,0.5)$ in (b) and at $(1,1,1)$ in (c) show the triplet $\left(\left|\ddot{\gamma}_{12}(\boldsymbol{\omega})\right|,\left|\ddot{\gamma}_{13}(\boldsymbol{\omega})\right|,\left|\ddot{\gamma}_{23}(\boldsymbol{\omega})\right|\right)$ which simultaneously maximizes all of its three elements. 


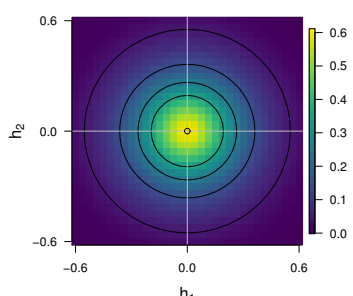

(a)

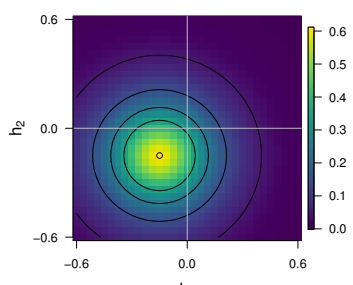

(d)

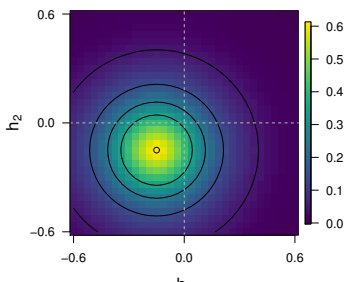

(g)

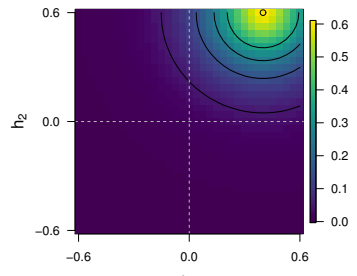

(j)

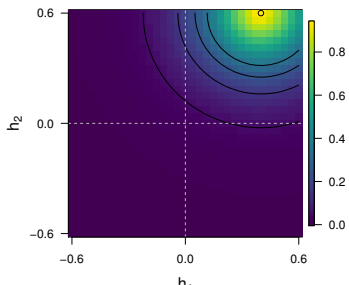

(m)

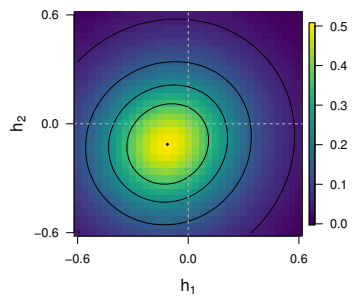

(p)

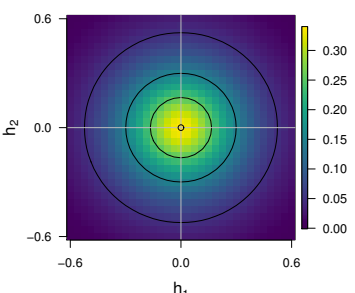

(b)

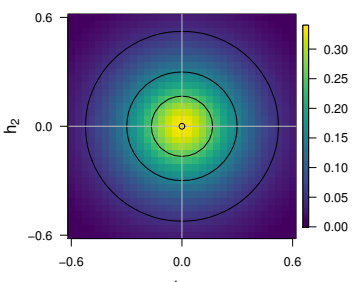

(e)

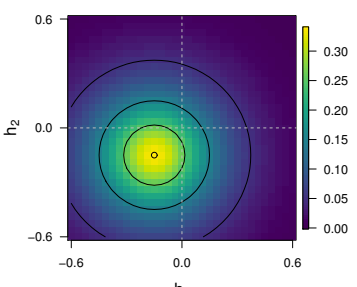

(h)

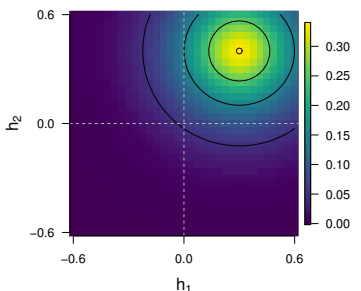

(k)

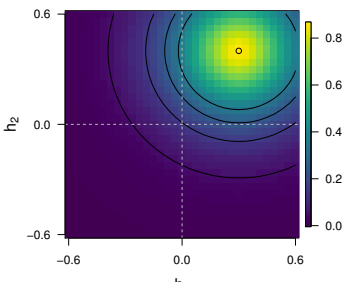

(n)

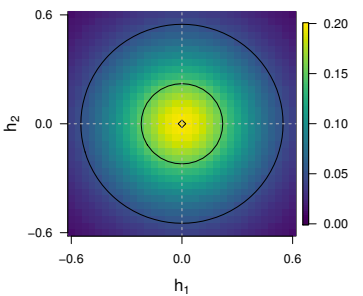

(q)

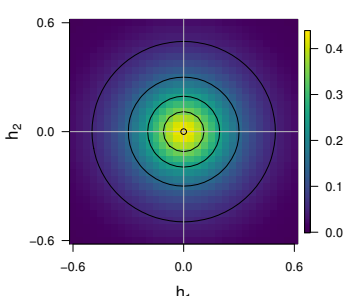

(c)

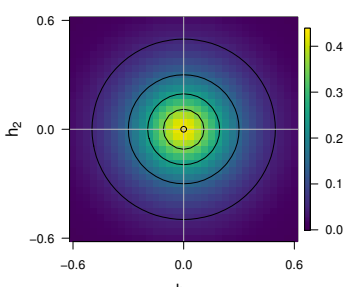

(f)

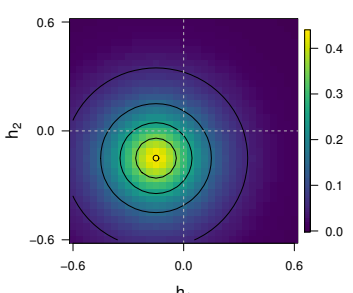

(i)

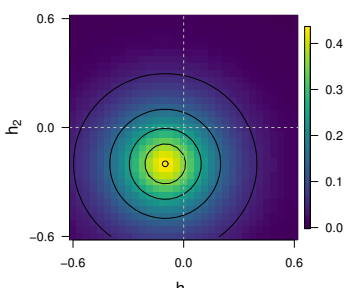

(1)

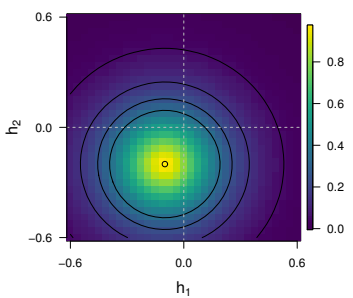

(o)

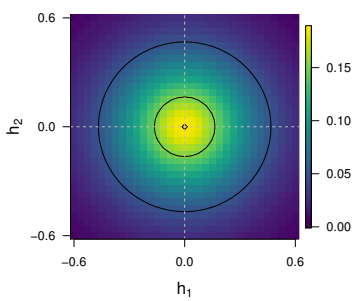

(r)

Figure 2: True cross-covariances for Case 1 ((a), (b), (c)), Case 2 ((d), (e), (f)), Case 3 $((\mathrm{g}),(\mathrm{h}),(\mathrm{i}))$, Case $4((\mathrm{j}),(\mathrm{k}),(\mathrm{l}))$, Case $5((\mathrm{~m}),(\mathrm{n}),(\mathrm{o}))$ and Case $6((\mathrm{p}),(\mathrm{q}),(\mathrm{r}))$. Plots in first, second and third columns represents $\operatorname{Cov}\left\{X_{1}(\mathbf{s}), X_{2}(\mathbf{s}+\mathbf{h})\right\}, \operatorname{Cov}\left\{X_{1}(\mathbf{s}), X_{3}(\mathbf{s}+\mathbf{h})\right\}$ and $\operatorname{Cov}\left\{X_{2}(\mathbf{s}), X_{3}(\mathbf{s}+\mathbf{h})\right\}$, respectively, for $\mathbf{h}=\left(h_{1}, h_{2}\right),-0.6 \leq h_{1}, h_{2} \leq 0.6$. 


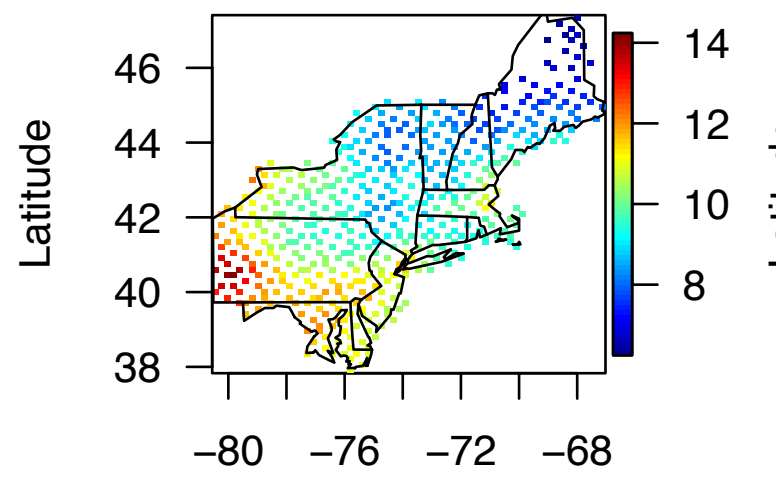

Longitude

(a)

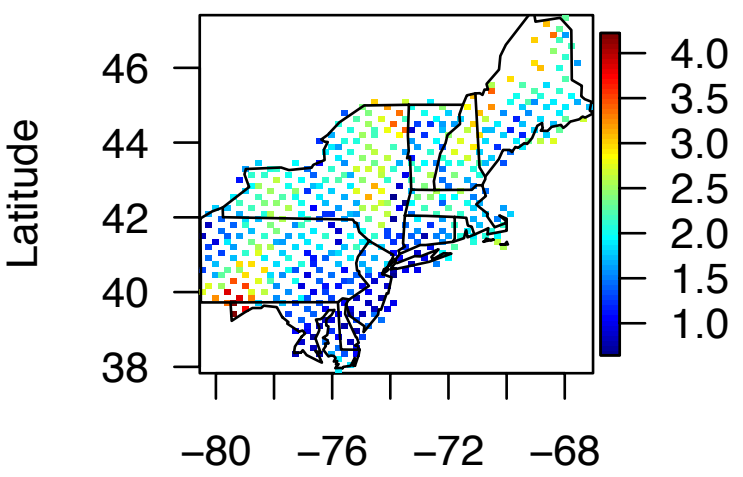

Longitude

(b)

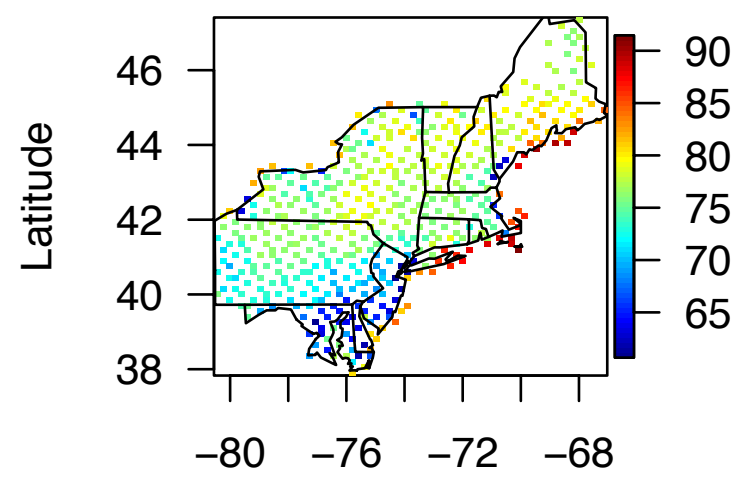

Longitude

(c)

Figure 3: Particulate matter concentrations $\left(\mathrm{PM}_{2.5}\right)$ (a), wind speed at 1000mb (WS) (b), and relative humidity at $2 \mathrm{~m}(\mathrm{RH})(\mathrm{c})$ over the North-Eastern climatic region of the United States. 
Table 2: Goodness-of-fit summary in terms average and standard error (SE) of maximized log-likelihood ( $\log \mathrm{L})$ and AIC values over 100 replicates. The bold values under each simulation case represent the maximum LogL and the minimum AIC, which corresponds to the best model under the respective simulation cases. In terms of AIC, Cases 2, 3 and 6 indicate the outstanding performance by the proposed model ${ }_{1} \mathbf{C}^{\mathrm{a}}(\mathbf{h})$. For Case 1 , the models ${ }_{\mathrm{k}} \mathbf{C}^{\mathrm{a}}(\mathbf{h}),{ }_{\mathrm{k}} \mathbf{C}^{\mathrm{LZ}}(\mathbf{h})$ and ${ }_{\mathrm{k}} \mathbf{C}(\mathbf{h})$ exhibit qualitatively equivalent performance in terms of $\log \mathrm{L}$, for $\mathrm{k}=1,2$, however, in terms $\mathrm{AIC},{ }_{\mathrm{k}} \mathbf{C}(\mathbf{h})$ outperformed ${ }_{\mathrm{k}} \mathbf{C}^{\mathrm{a}}(\mathbf{h})$ and ${ }_{\mathrm{k}} \mathbf{C}^{\mathrm{LZ}}(\mathbf{h})$, for $\mathrm{k}=1,2$. Cases $4-5$ indicate the best performance in terms of AIC by the model ${ }_{1} \mathbf{C}^{\mathrm{LZ}}(\mathbf{h})$.

\begin{tabular}{|c|c|c|c|c|c|c|c|}
\hline \multirow{2}{*}{ Case } & \multirow{2}{*}{ Criterion } & \multicolumn{6}{|c|}{ Candidate models } \\
\hline & & ${ }_{1} \mathbf{C}^{\mathrm{a}}(\mathbf{h})$ & ${ }_{1} \mathbf{C}^{\mathrm{LZ}}(\mathbf{h})$ & ${ }_{1} \mathbf{C}(\mathbf{h})$ & ${ }_{2} \mathbf{C}^{\mathrm{a}}(\mathbf{h})$ & ${ }_{2} \mathrm{C}^{\mathrm{LZ}}(\mathbf{h})$ & ${ }_{2} \mathbf{C}(\mathbf{h})$ \\
\hline \multirow{4}{*}{1} & Average(LogL) & 1451.9 & 1452.9 & 1451.0 & 1241.7 & 1241.7 & 1239.1 \\
\hline & $\mathrm{SE}(\log \mathrm{L})$ & 24.6 & 24.6 & 24.6 & 30.1 & 29.8 & 29.9 \\
\hline & Average(AIC) & -2871.7 & -2877.8 & -2881.9 & -2455.5 & -2459.4 & -2462.3 \\
\hline & $\mathrm{SE}(\mathrm{AIC})$ & 49.2 & 49.2 & 49.2 & 60.1 & 59.5 & 59.9 \\
\hline \multirow{4}{*}{2} & Average(LogL) & 1513.1 & 1411.6 & 1382.8 & 1243.4 & 1193.1 & 1169.6 \\
\hline & $\mathrm{SE}(\log \mathrm{L})$ & 24.5 & 29.4 & 27.9 & 34.1 & 35.5 & 34.5 \\
\hline & Average(AIC) & -2994.2 & -2795.2 & -2745.5 & -2458.9 & -2362.3 & -2323.3 \\
\hline & SE(AIC) & 49.0 & 58.9 & 55.9 & 68.2 & 70.9 & 68.9 \\
\hline \multirow{4}{*}{3} & Average(LogL) & 1472.1 & 1399.7 & 1303.8 & 1222.9 & 1188.6 & 1124.2 \\
\hline & $\mathrm{SE}(\log \mathrm{L})$ & 24.4 & 27.2 & 25.0 & 32.0 & 32.6 & 31.1 \\
\hline & Average(AIC) & -2912.2 & -2771.4 & -2587.5 & -2417.7 & -2353.3 & -2232.4 \\
\hline & SE(AIC) & 48.9 & 54.4 & 50.0 & 64.1 & 65.2 & 62.3 \\
\hline \multirow{4}{*}{4} & Average $(\log L)$ & 1356.1 & 1355.9 & 1303.7 & 1161.4 & 1161.3 & 1123.6 \\
\hline & $\mathrm{SE}(\log \mathrm{L})$ & 24.7 & 24.7 & 26.0 & 32.4 & 32.2 & 32.8 \\
\hline & Average(AIC) & -2680.1 & -2683.9 & -2587.3 & -2294.8 & -2298.6 & -2231.3 \\
\hline & SE(AIC) & 49.5 & 49.5 & 52.0 & 64.7 & 64.4 & 65.7 \\
\hline \multirow{4}{*}{5} & Average $(\operatorname{LogL})$ & 1664.0 & 1981.1 & 1304.5 & 1305.1 & 1344.9 & 1123.0 \\
\hline & $\mathrm{SE}(\log \mathrm{L})$ & 160.7 & 46.2 & 32.8 & 38.9 & 56.7 & 36.4 \\
\hline & Average(AIC) & -3296.1 & -3934.2 & -2588.9 & -2582.2 & -2665.7 & -2230.0 \\
\hline & SE(AIC) & 321.4 & 92.4 & 65.5 & 77.8 & 113.4 & 72.8 \\
\hline \multirow{4}{*}{6} & Average(LogL) & 2228.4 & 2219.8 & 2215.5 & 2104.2 & 2099.1 & 2096.6 \\
\hline & $\mathrm{SE}(\log \mathrm{L})$ & 25.6 & 26.0 & 24.1 & 29.4 & 29.5 & 27.6 \\
\hline & Average(AIC) & -4424.9 & -4414.6 & -4411.1 & -4180.3 & -4174.2 & -4177.2 \\
\hline & SE(AIC) & 51.3 & 52.0 & 48.2 & 58.7 & 59.0 & 55.1 \\
\hline
\end{tabular}


Table 3: Prediction performance summary in terms of percentage reduction of average $(\Delta$.A) and standard error $(\Delta . \mathrm{SE})$ of MSPE and mLogS over 100 replicates, with ${ }_{1} \mathbf{C}(\mathbf{h})$ as the base case, for all the candidate models under the six simulation cases. The bold values under each simulation case represent the maximum percentage reductions of averaged score which corresponds to the best model under the respective simulation cases. The proposed model ${ }_{1} \mathbf{C}^{\mathrm{a}}(\mathbf{h})$ produces highest percentage reductions of averaged scores among all the other candidate models for Cases 2-4, 6 and very close to the best competitor for Case 1. For Case $5,{ }_{1} \mathbf{C}^{\mathrm{LZ}}(\mathbf{h})$ produces the best scores.

\begin{tabular}{|c|c|c|c|c|c|c|}
\hline \multirow{2}{*}{ Case } & \multirow{2}{*}{$\begin{array}{l}\text { Percentage } \\
\text { reduction }\end{array}$} & \multicolumn{5}{|c|}{ Candidate models } \\
\hline & & ${ }_{1} \mathbf{C}^{\mathrm{a}}(\mathbf{h})$ & ${ }_{1} \mathbf{C}^{\mathrm{LZ}}(\mathbf{h})$ & ${ }_{2} \mathbf{C}^{\mathrm{a}}(\mathbf{h})$ & ${ }_{2} \mathbf{C}^{\mathrm{LZ}}(\mathbf{h})$ & ${ }_{2} \mathbf{C}(\mathbf{h})$ \\
\hline \multirow{4}{*}{1} & $\triangle . \mathrm{A}(\mathrm{MSPE})$ & $0.49 \%$ & $0.54 \%$ & $-28.16 \%$ & $-28.16 \%$ & $-30.36 \%$ \\
\hline & $\triangle . \mathrm{SE}(\mathrm{MSPE})$ & $1.98 \%$ & $2.12 \%$ & $-83.88 \%$ & $-82.08 \%$ & $-92.11 \%$ \\
\hline & $\Delta . \mathrm{A}(\mathrm{mLogS})$ & $0.06 \%$ & $0.12 \%$ & $-9.07 \%$ & $-9.09 \%$ & $-9.55 \%$ \\
\hline & $\Delta . \mathrm{SE}(\mathrm{mLogS})$ & $4.84 \%$ & $3.08 \%$ & $-18.97 \%$ & $-17.65 \%$ & $-24.91 \%$ \\
\hline \multirow{4}{*}{8} & $\Delta . \mathrm{A}(\mathrm{MSPE})$ & $38.59 \%$ & $22.09 \%$ & $0.21 \%$ & $-7.83 \%$ & $-34.47 \%$ \\
\hline & $\Delta . \mathrm{SE}(\mathrm{MSPE})$ & $37.94 \%$ & $20.01 \%$ & $-63.8 \%$ & $-71.81 \%$ & $-107.37 \%$ \\
\hline & $\Delta . \mathrm{A}(\mathrm{mLog} \mathrm{S})$ & $10.80 \%$ & $1.10 \%$ & $-4.85 \%$ & $-9.46 \%$ & $-11.30 \%$ \\
\hline & $\Delta . \mathrm{SE}(\mathrm{mLog} \mathrm{S})$ & $2.91 \%$ & $0.28 \%$ & $-31.38 \%$ & $-24.81 \%$ & $-21.21 \%$ \\
\hline \multirow{4}{*}{3} & $\Delta . \mathrm{A}(\mathrm{MSPE})$ & $37.26 \%$ & $29.28 \%$ & $10.90 \%$ & $7.00 \%$ & $-19.90 \%$ \\
\hline & $\Delta . \mathrm{SE}(\mathrm{MSPE})$ & $30.87 \%$ & $24.41 \%$ & $-30.34 \%$ & $-34.30 \%$ & $-64.72 \%$ \\
\hline & $\Delta . \mathrm{A}(\mathrm{mLog} \mathrm{S})$ & $12.92 \%$ & $7.12 \%$ & $-0.43 \%$ & $-3.21 \%$ & $-8.66 \%$ \\
\hline & $\Delta . \mathrm{SE}(\mathrm{mLog} \mathrm{S})$ & $-13.25 \%$ & $-4.83 \%$ & $-40.56 \%$ & $-44.9 \%$ & $-44.71 \%$ \\
\hline \multirow{4}{*}{4} & $\Delta . \mathrm{A}(\mathrm{MSPE})$ & $\mathbf{6 . 3 8} \%$ & $6.12 \%$ & $-13.70 \%$ & $-13.68 \%$ & $-19.24 \%$ \\
\hline & $\Delta . \mathrm{SE}(\mathrm{MSPE})$ & $9.98 \%$ & $8.18 \%$ & $-51.34 \%$ & $-50.46 \%$ & $-48.78 \%$ \\
\hline & $\Delta . \mathrm{A}(\mathrm{mLog} \mathrm{S})$ & $3.21 \%$ & $3.15 \%$ & $-6.32 \%$ & $-6.38 \%$ & $-8.67 \%$ \\
\hline & $\Delta . \mathrm{SE}(\mathrm{mLog} \mathrm{S})$ & $8.34 \%$ & $8.30 \%$ & $-13.14 \%$ & $-12.64 \%$ & $-10.30 \%$ \\
\hline \multirow{4}{*}{5} & $\Delta . \mathrm{A}(\mathrm{MSPE})$ & $7.97 \%$ & $34.35 \%$ & $-14.08 \%$ & $-1.16 \%$ & $-22.00 \%$ \\
\hline & $\Delta . \mathrm{SE}(\mathrm{MSPE})$ & $-14.53 \%$ & $21.03 \%$ & $-79.47 \%$ & $-73.91 \%$ & $-80.06 \%$ \\
\hline & $\Delta . \mathrm{A}(\mathrm{mLog} \mathrm{S})$ & $21.85 \%$ & $37.54 \%$ & $3.51 \%$ & $4.20 \%$ & $-8.89 \%$ \\
\hline & $\Delta . \mathrm{SE}(\mathrm{mLog} \mathrm{S})$ & $-271.80 \%$ & $-5.48 \%$ & $-36.44 \%$ & $-96.87 \%$ & $-21.82 \%$ \\
\hline \multirow{4}{*}{6} & $\Delta . \mathrm{A}(\mathrm{MSPE})$ & $\mathbf{3 . 8 8} \%$ & $-0.25 \%$ & $-13.47 \%$ & $-17.11 \%$ & $-18.27 \%$ \\
\hline & $\triangle . \mathrm{SE}(\mathrm{MSPE})$ & $-5.07 \%$ & $-12.89 \%$ & $-85.15 \%$ & $-93.81 \%$ & $-86.58 \%$ \\
\hline & $\Delta . \mathrm{A}(\mathrm{mLog} \mathrm{S})$ & $0.51 \%$ & $-0.01 \%$ & $-3.42 \%$ & $-3.77 \%$ & $-3.93 \%$ \\
\hline & $\Delta . \mathrm{SE}(\mathrm{mLog} \mathrm{S})$ & $-3.31 \%$ & $-4.89 \%$ & $-21.64 \%$ & $-21.87 \%$ & $-16.74 \%$ \\
\hline
\end{tabular}


Table 4: Maximum likelihood estimates (MLE), standard error (SE), and time to fit the model (in seconds) for ${ }_{3} \mathbf{C}^{\mathrm{a}}(\mathbf{h}),{ }_{3} \mathbf{C}^{\mathrm{LZ}}(\mathbf{h})$ and ${ }_{3} \mathbf{C}(\mathbf{h})$, based on the observed trivariate field $\mathbf{Y}$.

\begin{tabular}{ccccccc}
\hline \hline Candidate model & \multicolumn{2}{c}{${ }_{3} \mathbf{C}(\mathbf{h})$} & \multicolumn{2}{c}{${ }_{3} \mathbf{C}^{\mathrm{a}}(\mathbf{h})$} & \multicolumn{2}{c}{${ }_{3} \mathbf{C}^{\mathrm{LZ}}(\mathbf{h})$} \\
\hline Time & 2136.7 seconds & \multicolumn{2}{c}{3612.8 seconds } & \multicolumn{2}{c}{4281.1 seconds } \\
\hline Parameters & MLE & SE & MLE & SE & MLE & SE \\
$a$ & $5.82 \times 10^{-3}$ & $7.50 \times 10^{-7}$ & $5.82 \times 10^{-3}$ & $7.50 \times 10^{-7}$ & $5.82 \times 10^{-3}$ & $7.50 \times 10^{-7}$ \\
$\nu_{11}$ & 1.19 & 0.07 & 1.19 & 0.07 & 1.19 & 0.07 \\
$\sigma_{1}$ & 0.93 & 0.06 & 0.93 & 0.06 & 0.93 & 0.06 \\
$\nu_{22}$ & 0.87 & 0.19 & 0.87 & 0.19 & 0.87 & 0.19 \\
$\sigma_{2}$ & 1.01 & 0.07 & 1.01 & 0.07 & 1.01 & 0.07 \\
$\nu_{33}$ & 0.94 & 0.49 & 0.94 & 0.49 & 0.94 & 0.49 \\
$\delta_{1}$ & $9.76 \times 10^{-10}$ & $7.92 \times 10^{-10}$ & $9.76 \times 10^{-10}$ & $7.92 \times 10^{-10}$ & $9.76 \times 10^{-10}$ & $7.92 \times 10^{-10}$ \\
$\delta_{2}$ & 0.22 & 0.04 & 0.22 & 0.04 & 0.22 & 0.04 \\
$\delta_{3}$ & 0.47 & 0.08 & 0.47 & 0.08 & 0.47 & 0.08 \\
$\beta_{12}$ & -0.27 & 0.06 & -0.32 & 0.07 & -0.35 & 0.21 \\
$\beta_{13}$ & -0.24 & 0.08 & 0.44 & 0.09 & 0.47 & 0.10 \\
$\beta_{23}$ & 0.17 & 0.10 & 0.59 & 0.14 & -0.07 & 0.15 \\
$\boldsymbol{\ell}_{12}$ & -- & -- & $(9.66,10.17)$ & $(5.54,13.96)$ & -- & -- \\
$\boldsymbol{\ell}_{13}$ & -- & -- & $(-210.58,356.77)$ & $(14.75,10.03)$ & -- & -- \\
$\ell_{23}$ & -- & -- & $(793.38,174.18)$ & $(17.05,16.56)$ & -- & -- \\
$\ell_{2}$ & -- & -- & -- & -- & $(10.28,7.32)$ & $(11.93,103.23)$ \\
$\boldsymbol{\ell}_{3}$ & -- & -- & - & $(-199.98,-362.08)$ & $12.43,16.39$ \\
\hline
\end{tabular}

Table 5: Model comparison in terms of LogL, AIC, MSPE, and mLogS for ${ }_{3} \mathbf{C}^{\mathrm{a}}(\mathbf{h}),{ }_{3} \mathbf{C}^{\mathrm{LZ}}(\mathbf{h})$ and ${ }_{3} \mathbf{C}(\mathbf{h})$, based on the observed trivariate field $\mathbf{Y}$. The proposed model ${ }_{3} \mathbf{C}^{\mathrm{a}}(\mathbf{h})$ produces the highest (shown in bold) LogL and lowest values (shown in bold) of AIC, MSPE and mLogS.

\begin{tabular}{ccccc}
\hline \hline Candidate model & LogL & AIC & MSPE & LogS \\
\hline${ }_{3} \mathbf{C}^{\mathrm{a}}(\mathbf{h})$ & $\mathbf{- 1 1 0 2 . 9}$ & $\mathbf{2 2 4 3 . 7}$ & $\mathbf{0 . 3 1 3}$ & $\mathbf{1 . 0 6 1}$ \\
\hline${ }_{3} \mathbf{C}^{\mathrm{LZ}}(\mathbf{h})$ & -1109.8 & 2253.7 & 0.317 & 1.070 \\
\hline${ }_{3} \mathbf{C}(\mathbf{h})$ & -1118.7 & 2263.4 & 0.322 & 1.078 \\
\hline
\end{tabular}




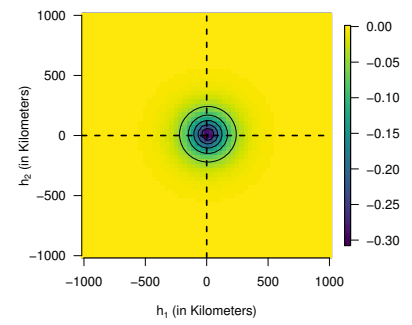

(a)

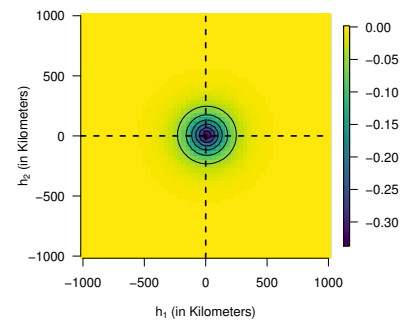

(e)

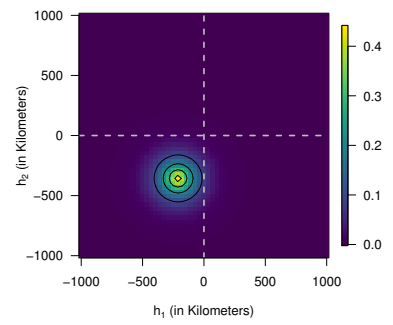

(b)

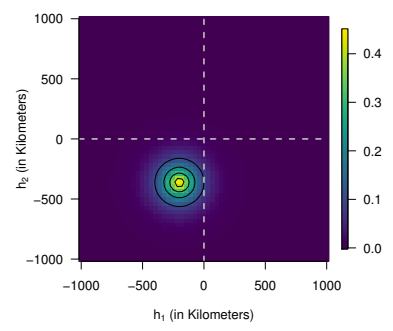

(f)

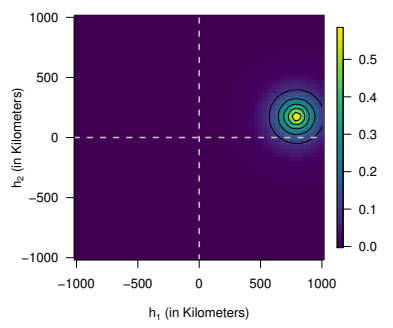

(c)

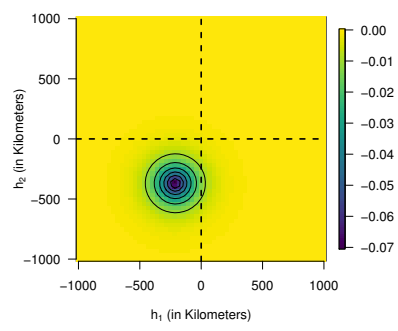

(g)

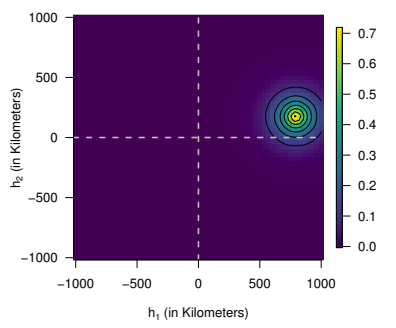

(d)

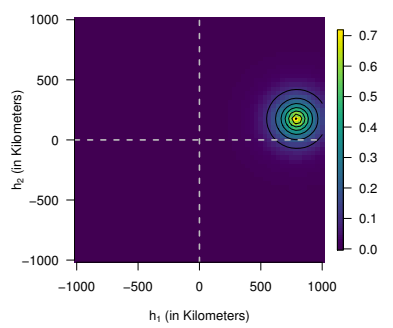

(h)

Figure 4: (a) and (e) show the estimated cross-correlation function $\operatorname{Cor}\left\{Y_{1}(\mathbf{s}), Y_{2}(\mathbf{s}+\mathbf{h})\right\}$ by using models ${ }_{3} \mathbf{C}^{\mathrm{a}}(\mathbf{h})$ and ${ }_{3} \mathbf{C}^{\mathrm{LZ}}(\mathbf{h})$, respectively. (b) and (f) show the estimated crosscorrelation function $\operatorname{Cor}\left\{Y_{1}(\mathbf{s}), Y_{3}(\mathbf{s}+\mathbf{h})\right\}$ by using models ${ }_{3} \mathbf{C}^{\mathrm{a}}(\mathbf{h})$ and ${ }_{3} \mathbf{C}^{\mathrm{LZ}}(\mathbf{h})$, respectively. (c) and (g) show the estimated cross-correlation function $\operatorname{Cor}\left\{Y_{2}(\mathbf{s}), Y_{3}(\mathbf{s}+\mathbf{h})\right\}$ by using models ${ }_{3} \mathbf{C}^{\mathrm{a}}(\mathbf{h})$ and ${ }_{3} \mathbf{C}^{\mathrm{LZ}}(\mathbf{h})$, respectively. (d) and (h) show the estimated cross-correlation function $\operatorname{Cor}\left\{Y_{2}(\mathbf{s}), Y_{3}(\mathbf{s}+\mathbf{h})\right\}$ from the bivariate fit of the model ${ }_{3} \mathbf{C}^{\mathrm{a}}(\mathbf{h})$ or equivalently ${ }_{3} \mathrm{C}^{\mathrm{LZ}}(\mathbf{h})$. 